\title{
Catalogue of the Tachinidae of Egypt (Diptera: Oestroidea)
}

\author{
Magdi S. El-Hawagry(D
}

\begin{abstract}
Tachinid flies are an important group of parasitoids in their larval stage, and all their hosts are of the Arthropoda, almost exclusively other insects, including important insect pests in agriculture and forestry. All known Egyptian taxa of the family Tachinidae are systematically catalogued. Synonymies, type localities, type depositories, world distributions by biogeographic realm(s) and country, Egyptian localities, and dates of collection are provided. A total of 72 tachinid species belonging to 42 genera, 15 tribes, and 4 subfamilies has been treated.
\end{abstract}

Keywords: Tachinid flies, Egyptian taxa, World distribution, Egyptian localities, Dates of collection

\section{Background}

Tachinidae are a large and cosmopolitan family of flies within the superfamily Oestroidea. It is the second largest family in the order Diptera (Irwin et al. 2003), with some 1500 recognized genera (O'Hara 2016) and more than 8500 described species (O'Hara 2013) worldwide. However, the estimated true diversity of the family is probably double the number of the currently known species, making this family a good candidate for the first largest family of Diptera (O'Hara, 2008 and 2013 and Pape et al. 2011).

Tachinid flies range in size from 2 to $20 \mathrm{~mm}$, and most of them are larger than a house fly and noticeably more bristly, especially on thoracic dorsum and third to fifth abdominal segments; however, across the family there is a tremendous variety of shapes, colors, and degree of bristling. In addition to features shared with other families of the super family Oestroidea (Rhinophoridae, Sarcophagidae, Calliphoridae, and Oestridae), adult tachinids can be recognized by the developed postscutellum (a rounded bulge below the hind part of the thoracic dorsum) (O'Hara 2008). The family is divided into four subfamilies: Dexiinae, Exoristinae, Phasiinae, and Tachininae. All four subfamilies are represented in Egypt as shown in the present results.

Tachinid flies are nectar or honeydew feeders as adults and are effective pollinators (Tooker et al. 2006). However, they are an important group of parasitoids in their larval stage, and all their hosts are of the phylum Arthropoda, almost exclusively other insects, including insect pests in

Correspondence: elhawagry@gmail.com

Entomology Department, Faculty of Science, Cairo University, Giza, Egypt agriculture and forestry. They typically parasitize phytophagous larvae of Lepidoptera and Coleoptera or nymphs of Hemiptera and Orthoptera. Consequently, tachinid flies have been successfully applied in programs of biological control against different insect pests (Stireman et al. 2006; O'Hara 2008 and Cerretti and Tschorsnig 2010).

No comprehensive taxonomic studies on the family Tachinidae have been carried out in Egypt before. Only one published study, Steyskal and El-Bialy (1967), listed the Egyptian species of dipterous families including Tachinidae based on literature and specimens deposited in the main Egyptian insect collections. However, despite its importance, the list included only the names of species within the family ordered alphabetically without referring to their suprageneric classification or to other important data such as original descriptions, type localities, synonymies, world distribution, type depositories, and local distribution. Another study, an unpublished Ph.D. thesis by Dr. Fathy Negm (Plant Protection Institute, Ministry of Agriculture) was completed in 1987 and reviewed the taxonomy of Egyptian Tachinidae. The author mainly adopted the classification of the Palaearctic catalogue of Herting (1984).

All missing data and deficiencies in previous Egyptian studies have been perfected with an updated taxonomy of the Egyptian Tachinidae in the present catalogue.

The present study is one in a series of planned studies on different Egyptian dipteran families aiming to catalogue the order Diptera as a whole in Egypt.

Egypt, as a part of the Great Desert Belt, is characterized by a warm and almost rainless climate (El-Hawagry 2017). 
Ecologists divide Egypt into eight ecological zones: Coastal Strip, Lower Nile Valley and Delta, Upper Nile Valley, Fayoum, Eastern Desert, Western Desert, Sinai, and Gebel Elba (Fig. 1). These zones have a greater affiliation to the Palaearctic Region except for the Gebel Elba, the southeastern triangle of Egypt, which has a greater affiliation to the Afrotropical Region (El-Hawagry and Gilbert 2014).

\section{Material and methods}

The present catalogue is based mainly on specimens collected by the author and his co-workers from different Egyptian localities, in addition to data compiled from specimens preserved in the main Egyptian insect collections, especially EFC and PPDD. A great deal of information, including synonymies and distributional data is obtained from relevant literature and website databases as well. Of the most important literature reviewed are Kugler (1980a); Herting (1984); Cerretti and Freidberg 2009; O'Hara et al. (2009); O'Hara and Cerretti (2016); and Pape and Thompson (2017).

\section{Scope}

This catalogue treats all names of taxa of the family Tachinidae recorded from Egypt.

\section{Arrangement of taxa}

All taxa are arranged alphabetically. Old world synonyms, including all available and unavailable names, are chronologically listed.

\section{Typographical treatment of names}

Family-group headings are centered and written in bold uppercase letters. Genus-group headings are left-justified

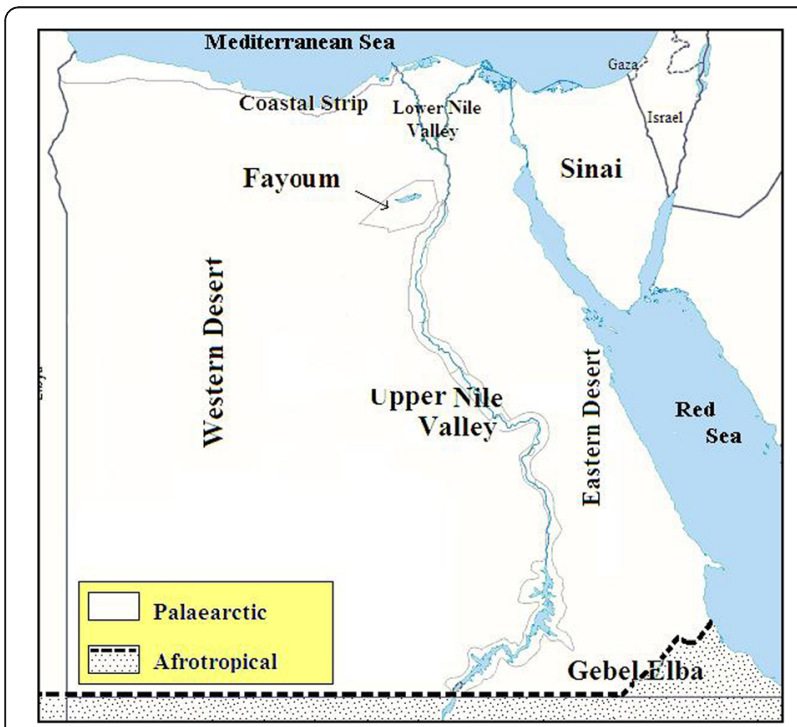

Fig. 1 Map of Egypt showing the ecological zones (taken from ElHawagry and Gilbert 2014) and written in bold uppercase letters. Species-group headings are left-justified and written in bold italicized lowercase letters except the first letter which is written uppercase. Authorship of genera, subgenera, and species are written in lowercase letters except the first letter which is written in uppercase. Taxonomically valid genus-group names (senior synonyms) are listed again in bold italicized lowercase letters (except the first uppercase letter) and left-justified under the headings followed by the reference including author, year, and journal pages. Taxonomically valid species-group names combined with their original genera (senior synonyms) are listed again in regular italicized lower case letters (except the first uppercase letter) and left-justified under the headings followed by the reference including author, year, and journal pages. Type species for all nomenclaturally available genus-group names are given after the reference line, followed by method of their fixation. For each genus and species-group name, associated synonyms are listed in a chronological order. They are written in regular italicized lowercase letters (except the first uppercase letter), followed by the reference and other data as in senior taxa. The rank of types that currently exist (e.g., syntype, holotype, lectotype) is given after the reference of each species-group taxon, followed by its location (depository) in museums, which is given as abbreviations in parenthesis. The type locality of each species-group taxon is given at the end after its type depository. Countries and islands of type localities are usually broken down to states, provinces, archipelagos, ecological zones, towns, and villages. These "sublocalities" are placed in parentheses after the main locality, e. g., "Egypt (Sinai)". The sublocalities may be more broken down to "smaller sublocalities". These "smaller sublocalities" are written after a colon following the sublocality, e.g., "Egypt (Sinai: Tor)".

\section{Distribution}

A complete world distribution by biogeographic realm(s) and country of each tachinid species, as is known from the literature, is listed in alphabetical order. Distribution localities preceded by a question mark (?) are considered questionable.

\section{Egyptian localities and dates of collection}

The Egyptian localities from which each tachinid species is collected and dates of collection are given to map a picture for the distribution and activity periods of the species in the different ecological zones of Egypt. Localities within each ecological zone are arranged alphabetically and written after a colon following the ecological zone, e.g., "Coastal Strip: Mariout". Spelling of locality names in Egypt almost follows El-Hawagry and Gilbert (2014) and El-Hawagry (2015 and 2017). 


\section{The catalogue}

This catalogue presents four tachinid subfamilies as represented in Egypt, and the number of taxa treated in the catalogue can be summarized in table.

\begin{tabular}{llll}
\hline Subfamily & Tribes & Genera & Species \\
\hline Dexiinae & 2 & 6 & 6 \\
Exoristinae & 5 & 21 & 37 \\
Phasiinae & 3 & 5 & 7 \\
Tachininae & 5 & 10 & 16 \\
Doubtful taxa & & & 6 \\
Total number & 15 & 42 & 72 \\
\hline
\end{tabular}

\section{Subfamily DEXIINAE}

Tribe DEXIINI

\section{Genus TRIXICEPS Villeneuve}

Trixiceps Villeneuve, 1936: 329. Type species:

Trixiceps aegyptiaca Villeneuve, 1936, by monotypy.

Trixiceps magnipalpis (Bezzi, 1922)

Paraprosena magnipalpis Bezzi, 1922: 154. Type(s) not located. Libya (Benghazi).

Trixiceps aegyptiaca Villeneuve, 1936: 329. Egypt (Ikingi Mariout).

Distribution: PA: Egypt, Europe, Libya.

Egyptian localities: Coastal Strip: Mariout, Mersa Matruh.

Dates of collection: April to August.

\section{Tribe VORIINI}

\section{Genus HYPOVORIA Villeneuve}

Hypovoria Villeneuve, 1913a: 510 (as subgenus of Voria Robineau-Desvoidy 1830). Type species: Voria (Hypovoria) hilaris Villeneuve, 1913, by monotypy.

Hypovoria hilaris (Villeneuve, 1913)

Voria (Hypovoria) hilaris Villeneuve, 1913a: 510. Holotype female (CNC). Tunisia (Sfax).

Distribution: PA: Canary Is., C. Asia (Kyrgyzstan, Tajikistan, Turkmenistan, Uzbekistan), China, Egypt, S. Europe (Albania, Bosnia and Herzegovina, Bulgaria, Croatia, France, Greece, Italy, Malta, Monaco, Montenegro, Macedonia, San Marino, Serbia, Slovenia), W. Europe (Andorra, Portugal (including Azores, excluding Madeira), Spain), Israel, Mongolia, Russia (E. Siberia), Tunisia, Transcaucasia.

Egyptian localities: Coastal Strip: El-Marg, Mariout.

Dates of collection: February to July.

Genus NANOPLAGIA Villeneuve

Nanoplagia Villeneuve, 1929a: 45. Type species: Plagia hilfii Strobl, 1902, by original designation.

Nanoplagia sinaica (Villeneuve, 1909)
Plagia hilfii sinaica Villeneuve in Hermann and Villeneuve, 1909: 157. Holotype female (CNC). Egypt (Sinai).

Distribution: PA: Algeria, China, Egypt, S. Europe (Albania, Bosnia and Herzegovina, Bulgaria, Croatia, France, Greece, Italy, Malta, Monaco, Montenegro, Macedonia, San Marino, Serbia, Slovenia), Israel, Kazakhstan, Morocco, Russia (W. Russia, E. Siberia), Transcaucasia.

Egyptian localities: Sinai:?

Dates of collection: April.

\section{Genus PERISCEPSIA Gistel}

Scopolia Robineau-Desvoidy, 1830: 268 (junior homonym of Scopolia Hübner). Type species: Musca carbonaria Panzer, 1798, by subsequent designation of Zetterstedt, 1844: 1239).

Periscepsia Gistel, 1848: x (nomen novum for Scopolia Robineau-Desvoidy, 1830).

Phoricheta Rondani, 1861a: 8 (nomen novum for Scopolia Robineau-Desvoidy, 1830).

Ramonda Robineau-Desvoidy, 1863a: 790. Type species: Ramonda fasciata Robineau-Desvoidy, 1863 (= Tachina spathulata Fallén, 1820) by original designation.

Subgenus PERISCEPSIA Gistel -Nominotypical subgenus

Periscepsia (Periscepsia) handlirschi (Brauer and von Bergenstamm, 1891)

Phorichaeta handlirschii Brauer and Bergenstamm, 1891: 52. Unspecified type(s) (NHMW). Italy (Trentino: Trafoi).

Note: The specific epithet was spelled handlirschii in the original description but was subsequently changed to handlirschi (O'Hara 2009).

Distribution: PA: China, Egypt, Israel, Europe.

Egyptian localities: Coastal Strip: Burg El-Arab, Mariout. Dates of collection: February to October.

Genus UCLESIA Girschner

Uclesia Girschner, 1901: 69. Type species: Uclesia fumipennis Girschner, 1901, by monotypy.

Uclesia petiolata (Villeneuve, 1929)

Nanoplagia petiolata Villeneuve, 1929a: 45. Holotype female (?EFC, not located). Egypt (Wadi Rishrash).

Distribution: PA: Algeria, Tunisia, Egypt, Russia, Tadzhikistan.

Egyptian localities: Eastern Desert: Wadi Rishrash.

Dates of collection: March.

Genus WAGNERIA Robineau-Desvoidy

Wagneria Robineau-Desvoidy, 1830: 126. Type species: Wagneria gagatea Robineau-Desvoidy, 1830, by monotypy.

Wagneria cunctans (Meigen, 1824)

Tachina cunctans Meigen, 1824: 419. Holotype male (MNHN). Germany. 
Wagneria riedeli Villeneuve, 1937: 405. Germany (Frankfurt).

Distribution: PA: Egypt, Europe, Israel.

Egyptian localities: Coastal Strip: El-Burg, Mariout, Nuzha. Eastern Desert: Suez Road. Lower Nile Valley and Delta: Dokki. Sinai: Ein Mouussa.

Dates of collection: January to July.

\section{Subfamily EXORISTINAE \\ Tribe ERYCIINI}

\section{Genus AMELIBAEA Mesnil}

Amelibaea Mesnil, 1955: 454 (as subgenus of Phebellia Robineau-Desvoidy, 1846). Type species: Parexorista tultschensis Brauer and Bergenstamm, 1891, by monotypy.

Amelibaea ?signifera (Villeneuve, 1929)

Exorista signifera Villeneuve, 1929a: 44. Holotype male (?EFC, not located). Egypt (Mariout).

Remarks: The identity of this species is not clear since the male holotype is apparently lost (Cerretti and Freidberg 2009).

Distribution: PA: Egypt, Israel (Cerretti and Freidberg 2009).

Egyptian localities: Coastal Strip: Mariout.

Dates of collection: January.

\section{Genus APLOMYA Robineau-Desvoidy}

Aplomya Robineau-Desvoidy, 1830: 184. Type species: Aplomya zonata Robineau-Desvoidy, 1830 (= Tachina confinis Fallén, 1820), by subsequent designation of Robineau-Desvoidy (1863a: 459, 460).

Prozenillia Villeneuve, 1916: 487. Type species: Prozenillia distans Villeneuve, 1916, by monotypy.

Wiedemanniomyia Townsend, 1933: 469. Туре species: Tachina metallica Wiedemann, 1824, by original designation.

Aplomyiella Mesnil, 1939: 31. Type species: Tricholyga impexa Villeneuve, 1916 (= Tachina metallica Wiedemann, 1824), by original designation.

Atricholyga Villeneuve, 1939: 9. Type species: Tricholyga impexa Villeneuve, 1916 (= Tachina metallica Wiedemann, 1824), by monotypy.

Aplomya confinis (Fallén, 1820)

Tachina confinis Fallén, 1820b: 32. Syntypes, males and females (NHRS and/or MZLU). Sweden (Gotland).

Phryxe zonata Robineau-Desvoidy, 1830: 159. France.

Phryxe servillei Robineau-Desvoidy, 1830: 159. France (Paris).

Aplomya nitens Robineau-Desvoidy, 1830: 185. France (Yonne: St. Sauveur).

Aplomya zonata Robineau-Desvoidy, 1830: 185. France (Paris).

Tachina bicingulata Zetterstedt, 1844: 1133. Sweden (Skäne: Esperod).
Exorista aenescens Macquart, 1850: 409. France (Lestrem).

Exorista longicornis Perris, 1852: 206. France (Grandes Landes).

Tachina bizonata Zetterstedt, 1859: 6119. Sweden (Skäne: Sjobo).

Distribution: AF: ?Malawi, Yemen. OR: Orien. China [see O'Hara and Cerretti 2016: 19]. PA: Canary Is., C. Asia (Kyrgyzstan, Tajikistan, Turkmenistan, Uzbekistan), Egypt, Europe, Israel, Japan, Korea, Kuril Is., Madeira, Mongolia, Pal. China [see O'Hara and Cerretti 2016: 19], Russia, Transcaucasia.

Egyptian localities: Lower Nile Valley and Delta: Abu Rawash, Koubba.

Dates of collection: February to April.

Aplomya metallica (Wiedemann, 1824)

Tachina metallica Wiedemann, 1824: 46. Lectotype male (ZMUC). India. [by fixation of Townsend (1933: 470)].

Tachina nigriventris Wiedemann, 1824: 43. India.

Tachina notata Wiedemann, 1830: 653. Sudan (Nubia Region [as "Nubien"]).

Tachina socia Wiedemann, 1830: 654. Sudan (Nubia Region [as "Nubien"]).

Phorocera eucalypta Loew, 1852: 659. Mozambique (Tete [as "Tette"]).

Parexorista laeviventris Wulp, 1893: 173. Indonesia (Jawa).

Tricholyga impexa Villeneuve, 1916: 494. D.R. Congo and South Africa (Eastern Cape).

Distribution: AF: Widespread. OR: Widespread. Palaearctic: Egypt, Israel, Japan, Pal. China [see O’Hara and Cerretti 2016: 19].

Egyptian localities: Upper Nile Valley: Asswan.

Dates of collection: Unknown.

\section{Genus CADURCIELLA Villeneuve}

Cadurciella Villeneuve, 1927: 120. Type species: Cadurciella rufipalpis Villeneuve, 1927, by monotypy.

Cadurciella tritaeniata (Rondani, 1859)

Exorista tritaeniata Rondani, 1859: 147. Unspecified type(?MZF). Italy (Parma).

Distribution: PA: Egypt, Europe (northwards to Scotland, S. Norway, Sweden, Japan.

Egyptian localities and dates of collection are not precisely known. Recorded by Steyskal and El-Bialy (1967: 48).

\section{Genus CESTONIA Rondani}

Cestonia Rondani, 1861a: 105. Type species: Cestonia cineraria Rondani, 1861, by monotypy.

Cestonia cineraria Rondani, 1861

Cestonia cineraria Rondani, 1861a: 106. Syntypes, males and females (MZF). Italy (Parma).

Distribution: PA: Egypt, Europe (Mediterranean, northwards to S. France, Switzerland, and Italy), Israel. 
Egyptian localities: Coastal Strip: Nuzha. Eastern Desert: Wadi Hoff. Lower Nile Valley and Delta: Ezbet El-Nakhl. Sinai: Wadi El-Tih.

Dates of collection: March to July.

Cestonia rutilans Villeneuve, 1929

Cestonia rutilans Villeneuve, 1929b: 102. Syntypes, 1 male and 1 female, not located. Egypt (Cairo).

Distribution: AF: Senegal, Yemen. PA: Egypt, Libya.

Egyptian localities and dates of collection are not precisely known.

\section{Genus CESTONIONERVA Villeneuve}

Cestonionerva Villeneuve, 1929a: 43. Type species: Conogaster petiolata Villeneuve, 1910, by subsequent designation of Townsend (1936: 137).

\section{Cestonionerva petiolata (Villeneuve, 1910)}

Conogaster petiolata Villeneuve in Becker, 1910: 144. Holotype female (NHMW, not located). Yemen (Socotra).

Cestonionerva proxima Villeneuve, 1929a: 43. Egypt (Dahshour).

Distribution: AF: U.A. Emirates, Yemen. PA: Canary Is., C. Asia (Kyrgyzstan, Tajikistan, Turkmenistan, Uzbekistan), Israel, Mongolia, North Africa, Pal. China [see O'Hara and Cerretti 2016: 19].

Egyptian localities: Lower Nile Valley and Delta: Dahshour.

Dates of collection: April.

\section{Genus DRINO Robineau-Desvoidy}

Drino Robineau-Desvoidy, 1863a: 250. Type species: Drino volucris Robineau-Desvoidy, 1863 (= Tachina lota Meigen, 1824), by original designation.

\section{Subgenus PALEXORISTA Townsend}

Palexorista Townsend, 1921: 134. Type species: Tachina succini Giebel, 1862 (as "Tichina succini Giebel"), by monotypy.

Prosturmina Mesnil, 1970b: 110 (as subgenus of Drino Robineau-Desvoidy). Type species: Sturmia vigilans Villeneuve, 1933 (= Sturmia pulchra Curran, 1927), by original designation.

Drino (Palexorista) gilva (Hartig, 1838)

Tachina gilva Hartig, 1838: 288. Syntypes, males and females, not located. Germany.

Drino aurora Mesnil, 1949: 15. Japan.

Distribution: PA: Egypt, Europe, Japan, Transcaucasia.

Egyptian localities and dates of collection are not precisely known. Recorded from Egypt by Steyskal and El-Bialy (1967: 48).

Drino (Palexorista) imberbis (Wiedemann, 1830)

Tachina imberbis Wiedemann, 1830: 317. Syntypes, males (ZMUH, lost). Egypt.

Sturmia zonata Curran, 1927b: 336. Uganda (Entebbe).

Distribution: AF: D.R. Congo, Kenya, Malawi, South Africa, Sudan, Tanzania, U.A. Emirates, Uganda, Yemen. PA: Canary Is., C. Asia (Kyrgyzstan, Tajikistan, Turkmenistan, Uzbekistan), Egypt, Iran, Israel, North Africa, Turkey, Turkmenia.
Egyptian localities and dates of collection are not precisely known.

Drino (Palexorista) inconspicua (Meigen, 1830)

Tachina inconspicua Meigen, 1830: 369. Unspecified types, females (MNHN). Germany (Berlin).

Tachina bimaculata Hartig, 1838: 286. Germany.

Tachina flavoscutellata Zetterstedt, 1844: 1042. Sweden.

Masicera cursitans Rondani, 1861a: 21. Italy (Parma).

Sturmia lophyri Robineau-Desvoidy, 1863b: 892.

Germany.

Drino inusta Mesnil, 1949: 25. Poland.

Distribution: PA: C. Asia (Kyrgyzstan, Tajikistan, Turkmenistan, Uzbekistan), China, Egypt, Europe, Russia (W. Russia, W. Siberia), Taiwan, Transcaucasia.

Egyptian localities and dates of collection are not precisely known.

Subgenus ZYGOBOTHRIA Mik

Zygobothria Mik, 1891: 193. Type species: Sturmia atropivora Robineau-Desvoidy, 1830 , by original designation.

Formosodoria Townsend, 1933: 475. Type species: Sturmia (Argyrophylax) dilabida Villeneuve, 1916 (= Meigenia ciliata van der Wulp, 1881), by original designation.

Drino (Zygobothria) atropivora (Robineau-Desvoidy, 1830)

Sturmia atropivora Robineau-Desvoidy, 1830: 171. Syntypes, males and females (MNHN). France.

Sturmia masakensis Curran, 1927c: 117. Uganda (Masaka).

Distribution: AF: Widespread. AU: Australia. OR: India, Indonesia, Laos, Malaysia, Orien. China [see O'Hara and Cerretti, 2016: 19], Ryukyu Is., Sri Lanka. PA: Algeria, Canary Is., C. Asia (Kyrgyzstan, Tajikistan, Turkmenistan, Uzbekistan), Egypt, all Europe (except British Is., Scand.), Israel, Japan, Pal. China [see O’Hara and Cerretti 2016: 19], Morocco, Russia, Transcaucasia, Tunisia.

Egyptian localities: Coastal Strip: Ramleh. Eastern Desert: Ismailia, Suez Road. Lower Nile Valley and Delta: El-Qubba, Dekernes, Maadi, Shubra.

Dates of collection: April to July.

\section{Genus THECOCARCELIA Townsend}

Thecocarcelia Townsend, 1933: 471. Type species: Argyrophylax pelmatoprocta Brauer and Bergenstamm, 1891 (= Masicera acutangulata Macquart, 1851), by original designation.

Thelycarcelia Townsend, 1933: 475. Type species: Thelycarcelia thrix Townsend, 1933 (= Sturmia sumatrana Baranov, 1932), by original designation.

Thecocarcelia acutangulata (Macquart, 1851)

Masicera acutangulata Macquart, 1851a: 478. Unspecified type, female (?MHNL). Switzerland (Chur [as "Coire"]). 
Masicera incedens Rondani, 1861a: 22. Italy (Parma).

Argyrophylax pelmatoprocta Brauer and Bergenstamm, 1891: 344. Austria (Bisamberg: nr. Vienna).

Distribution: AF: Widespread. PA: Palaearctic: Egypt, all Europe (except Turkey), Japan, Transcaucasia.

Egyptian localities and dates of collection are not precisely known.

\section{Tribe ETHILLINI}

\section{Genus ATYLOMYIA Brauer}

Atylomyia Brauer, 1898: 525. Type species: Atylomyia loewii Brauer, 1898, by monotypy.

Atylomyia albifrons Villeneuve, 1911

Atylomyia albifrons Villeneuve, 1911: 86. Lectotype female (CNC). Egypt (Helwan).

Atylomyia rungsi Mesnil, 1962: 778. Holotype. Morocco (Ait Melloul).

Distribution: PA: China, Egypt, Israel, W. Europe (Andorra, Portugal (including Azores, excluding Madeira), Spain).

Egyptian localities: Lower Nile Valley and Delta: Helwan.

Dates of collection: January and February.

\section{Tribe EXORISTINI}

Genus ALLOPROSOPAEA Villeneuve

Alloprosopaea Villeneuve, 1923: 89. Type species: Alloprosopaea efflatouni Villeneuve, 1923, by monotypy.

Alloprosopaea efflatouni Villeneuve, 1923

Alloprosopaea efflatouni Villeneuve, 1923: 90.

Unspecified type(s) (CNC). Egypt (Wadi Hoff).

Distribution: PA: Egypt, Israel.

Egyptian localities: Eastern Desert: Wadi Hoff. Lower Nile Valley and Delta: Dahshour.

Dates of collection: April.

Genus CHAETORIA Becker

Chaetoria Becker, 1908: 113. Type species: Chaetoria stylata Becker, 1908, by monotypy.

Clistorrhinia Bezzi in Bezzi and Lamb, 1926: 570.

Type species: Clistorrhinia aurifrons Bezzi, 1926, by monotypy.

\section{Chaetoria stylata Becker, 1908}

Chaetoria stylata Becker, 1908: 114. Lectotype female (ZMHB). Canary Islands (Tenerife).

Distribution: AF: Botswana, Mozambique, Nigeria, Senegal, U.A. Emirates, Yemen. PA: Algeria, Canary Is., C. Asia (Kyrgyzstan, Tajikistan, Turkmenistan, Uzbekistan), Egypt, Europe, Morocco, Tunisia.

Egyptian localities: Coastal Strip: Ramleh. Upper Nile Valley: Luxur.

Dates of collection: January to September.

\section{Genus CHETOGENA Rondani}

Salia Robineau-Desvoidy, 1830: 108 (junior homonym of Salia Hübner, 1818). Type species: Salia echinura
Robineau-Desvoidy, 1830 (= Tachina obliquata Fallén 1810), by subsequent designation of Robineau-Desvoidy (1863a: 553).

Chetogena Rondani, 1856: 68. Type species: Salia rondaniana Villeneuve, 1931.

Spoggosia Rondani, 1859: 182. Type species: Spoggosia occlusa Rondani, 1859 (=Tachina obliquata Fallén 1810), by monotypy.

Stomatomyia Brauer and Bergenstamm, 1889: 98. Type species: Chetogena filipalpis Rondani, 1859, by subsequent designation of Brauer (1893: 483).

Chetogena aegyptiaca (Villeneuve, 1923)

Tachinosalia aegyptiaca Villeneuve, 1923: 91. Holotype male (EFC). Egypt.

Distribution: PA: Egypt, Israel.

Egyptian localities: Coastal Strip: Mariout.

Dates of collection: March.

Chetogena cercosa (Kugler, 1980)

Chaetogena cercosa Kugler, 1980a: 31. Holotype male (TAU). Israel (Elat).

Distribution: AF: U. A. Emirates. PA: Egypt, Israel.

Egyptian localities: Sinai: Serabit El-Khadim.

Dates of collection: April.

Chetogena sinaica (Villeneuve, 1909)

Stomatomyia acuminata var. sinaica Villeneuve in Hermann and Villeneuve, 1909: 157. Holotype female (CNC). Egypt (Sinai).

Distribution: PA: Egypt, Israel.

Egyptian localities: Sinai: South Sinai.

Dates of collection: ?April.

\section{Genus EXORISTA Meigen}

Exorista Meigen, 1803: 280. Type species: Musca larvarum Linnaeus, 1758 , by monotypy.

Subgenus ADENIA Robineau-Desvoidy

Adenia Robineau-Desvoidy, 1863a: 1041. Type species: Tachina grisea Robineau-Desvoidy, 1830 (= Tachina rustica Fallén, 1810), by original designation.

Staegeria Robineau-Desvoidy, 1863a: 972 (junior homonym of Staegeria Rondani 1856). Type species: Tachina pratensis Robineau-Desvoidy, 1830, by original designation.

Chaetotachina Brauer and Bergenstamm, 1889: 98. Type species: Tachina rustica Fallén, 1810, by monotypy.

Exorista (Adenia) rustica (Fallén, 1810)

Tachina rustica Fallén, 1810: 264. Lectotype male (NHRS). Sweden (Skåne).

Distribution: PA: C. Asia (Kyrgyzstan, Tajikistan, Turkmenistan, Uzbekistan), China, Egypt, all Europe, M. East, Mongolia, Russia, Taiwan, Transcaucasia.

Egyptian localities: Eastern Desert: Wadi Hoff.

Dates of collection: March to June.

Subgenus EXORISTA Meigen -Nominotypical subgenus

Exorista Meigen, 1803: 280. Type species: Musca larvarum Linnaeus, 1758 (as "Musca larvarum Fabr."), by monotypy. 
Exorista (Exorista) fasciata (Fallén, 1820)

Tachina fasciata Fallén, 1820a: 5. Syntypes, males and females (NHRS and/or MZLU). Sweden (Skåne, Äsperöd [as "Esperöd"] and Abusa).

Chetogena segregata Rondani, 1859: 181. Italy (Parma and Sicily); Malta.

Distribution: NE: Yukon, introduced and established in Ontario, Québec and New England. OR: India. PA: China, Egypt, Europe, Japan, M. East, Mongolia, Russia, Taiwan, Transcaucasia.

Egyptian localities: Upper Nile Valley: Assiout.

Dates of collection: March.

Exorista (Exorista) larvarum (Linnaeus, 1758)

Musca larvarum Linnaeus, 1758: 596. Unspecified Type(s) (LSUK). Europe.

Tachina moreti Robineau-Desvoidy, 1853: 534. France (Auxerre).

Distribution: NE: New England, Ontario, Québec, Yukon. OR: India. PA: C. Asia (Kyrgyzstan, Tajikistan, Turkmenistan, Uzbekistan), China, all Europe, Japan, M. East, Mongolia, North Africa, Russia, Taiwan, Transcaucasia.

Egyptian localities: Coastal Strip: Alexandria, Mariout, Nuzha. Eastern Desert: Ismailia. Lower Nile Valley and Delta: Bahtim, Dessouk, Dokki, Embaba, Sakha, Tanta. Fayoum: ?. Sinai: El-Arish. Upper Nile Valley: Sohag.

Dates of collection: Throughout the year.

Subgenus PODOTACHINA Brauer and Bergenstamm

Podotachina Brauer and Bergenstamm, 1891: 350. Type species: Tachina sorbillans Wiedemann, 1830, by subsequent designation of Townsend (1916: 8).

Exorista (Podotachina) sorbillans (Wiedemann, 1830)

Tachina sorbillans Wiedemann, 1830: 311. Lectotype male (NHMW). Canary Islands (Tenerife).

Distribution: AF: Cameroon, Canary Is., D.R. Congo, Kenya, Malawi, Nigeria, Sierra Leone, Uganda. OR: India, Indonesia, Nepal, Orien. China [see O'Hara and Cerretti 2016: 19], Philippines, Ryukyu Is., Sri Lanka, Taiwan, Thailand, Vietnam. Australasian: Australia, N. Australasian. PA: C. Asia (Kyrgyzstan, Tajikistan, Turkmenistan, Uzbekistan), Egypt, Europe, Israel, Japan, Mongolia, China.

Egyptian localities and dates of collection are not precisely known. Recorded from Egypt by Steyskal and El-Bialy (1967: 49).

Subgenus PTILOTACHINA Brauer and
Bergenstamm

Ptilotachina Brauer and Bergenstamm, 1891: 350. Type species: Exorista florentina Herting, 1975, by fixation of O'Hara et al. (2009: 94) under Article 70.3.2 of the Code (ICZN, 1999).

Exorista (Ptilotachina) rutilans Mesnil, 1970

Exorista rutilans Mesnil, 1970a: 113. Holotype male (MNHN). Egypt (Sinai).
Distribution: PA: Egypt, Israel.

Egyptian localities: Sinai.

Dates of collection: April.

Exorista (Ptilotachina) xanthaspis (Wiedemann, 1830)

Tachina xanthaspis Wiedemann, 1830: 314. Syntypes, males and females (SMF). Sudan (Nubia Region [as "Nubien"]).

Tachina pyrrhocera Wiedemann, 1830: 314. Sudan (Nubia Region [as "Nubien"]).

Tachina fallax pseudofallax Villeneuve, 1920: 151. South Africa (Eastern Cape).

Larvaevora (Ptilotachina) fallax aethiopica

Rohdendorf, 1931: 348. Sudan (Wad Medani).

Distribution: AF: Madagascar, Seychelles, Sudan, U.A. Emirates, Yemen. AU: N. Australasian. OR: India, Indonesia, Orien. China [see O'Hara and Cerretti 2016: 19], Ryukyu Is., Taiwan. PA: C. Asia (Kyrgyzstan, Tajikistan, Turkmenistan, Uzbekistan), Egypt, all Europe (except British Is., Scand.), Israel, Mongolia, Pal. China [see O'Hara and Cerretti 2016: 19], Russia, Transcaucasia.

Egyptian localities: Lower Nile Valley and Delta: Abu Rawash. Sinai: St. Katherine.

Dates of collection: February to August. Upper Nile Valley: Asswan.

\section{Genus MACULOSALIA Mesnil}

Maculosalia Mesnil, 1946: 62 (as subgenus of Spoggosia Rondani 1859). Type species: Deuterammobia maculosa Villeneuve, 1909, by monotypy.

Maculosalia maculosa (Villeneuve, 1909)

Deuterammobia maculosa Villeneuve, 1909: 80. ?Holotype (?MNHN). Egypt (Sinai).

Distribution: PA: Egypt, Iran, Tunisia.

Egyptian localities: Sinai: ?

Dates of collection: Unknown.

\section{Tribe GONIINI}

Genus BLEPHARELLA Macquart

Blepharella Macquart, 1851b: 176. Type species: Blepharella lateralis Macquart, 1851, by original designation.

Blepharella versatilis (Villeneuve, 1910)

Sturmia versatilis Villeneuve, 1910a: 253. Unspecified type(s), 1 male (CNC). D.R. Congo.

Distribution: AF: D.R. Congo, Malawi, Nigeria, Sudan. PA: ?Egypt.

Note: Villeneuve (1913b: 29) recorded this species from Egypt, based on a male in $\mathrm{BMNH}$, however this record needs confirmation (O'Hara and Cerretti 2016).

Genus CLEMELIS Robineau-Desvoidy

Clemelis Robineau-Desvoidy, 1863a: 481. Type species: Zenillia ciligera Robineau-Desvoidy, 1830 (= Tachina pullata Meigen, 1824), by original designation. 
Clemelis apicalis Villeneuve, 1923

Clemelis apicalis Villeneuve, 1923: 92. Holotype male (?EFC, not located). Egypt [as "Lower Egypt"].

Distribution: PA: Egypt.

Egyptian localities: Coastal Strip: Mariout.

Dates of collection: April.

Genus GONIA Meigen

Salmacia Meigen, 1800: 38. Name suppressed by ICZN (1963: 339).

Gonia Meigen, 1803: 280. Type species: Gonia bimaculata Wiedemann, 1819, by subsequent designation of Sabrosky and Arnaud Jr (1965: 1075).

Reaumuria Robineau-Desvoidy, 1830: 79. Type species: Musca capitata De Geer, 1776, by subsequent designation of Robineau-Desvoidy (1863a: 733).

Pissemya Robineau-Desvoidy, 1851b: 318. Type species: Gonia atra Meigen, 1826, by monotypy.

Turanogonia Rohdendorf, 1924: 228. Type species: Turanogonia smimovi Rohdendorf, 1924 (= Gonia chinensis Wiedemann, 1824), by monotypy.

Asiogonia Rohdendorf, 1928: 98. Type species: Asiogonia asiatica Rohdendorf, 1928, by monotypy.

Chrysocerogonia Rohdendorf, 1928: 98 (as subgenus of Salmacia Meigen, 1800). Type species: Salmacia (Chrysocerogonia) ussuriensis Rohdendorf, 1928, by monotypy.

Eremogonia Rohdendorf, 1928: 98 (as subgenus of Salmacia Meigen, 1800). Type species: Salmacia (Eremogonia) desertorum Rohdendorf, 1928, by monotypy.

Gonia atra Meigen, 1826

Gonia atra Meigen, 1826: 7. Unspecified type(s) (MNHN). France.

Distribution: PA: C. Asia (Kyrgyzstan, Tajikistan, Turkmenistan, Uzbekistan), China, Egypt, Europe, Kazakhstan, Mongolia, Russia (Siberia), Transcaucasia.

Egyptian localities: Fayoum:? . Lower Nile Valley and Delta:

El-Marg, Ezbet El-Nakhl, Kafr Hakim, Kerdassa, Sentrees.

Dates of collection: February to October.

Gonia bimaculata Wiedemann, 1819

Gonia bimaculata Wiedemann, 1819: 25. Syntypes, females (NHMW, ZMUC). South Africa (Western Cape: Cape of Good Hope).

Gonia cilipeda Rondani, 1859: 35. Italy (Parma and Malta).

Gonia incerta Bigot, 1888: 10. (Tunisia. Tunis).

Salmacia cabrerae Mesnil, 1956: 523. Canary Is. (Hierro).

Distribution: AF: Widespread (excl. W. AF). OR: Orien. China [see O'Hara and Cerretti 2016: 19]. PA: Canary Is., C. Asia (Kyrgyzstan, Tajikistan, Turkmenistan, Uzbekistan), Europe, Israel, Madeira, North Africa, Pal. China [see O'Hara and Cerretti 2016: 19], Transcaucasia.

Egyptian localities: Coastal Strip: Abu Qir. Eastern Desert: Gebel Asfar, Ismailia, Wadi Hoff. Fayoum:
Kom Osheem. Lower Nile Valley and Delta: Abu Rawash, Borkash, El-Marg, Ezbet El-Nakhl, Kafr Hakim, Pyramids, Shubra, Tanta, Zaqaziq.

Dates of collection: February to September.

Gonia capitata (De Geer, 1776)

Musca capitata De Geer, 1776: 23. Syntypes, unspecified number and sex (?NHRS). Sweden.

Rhedia testacea Robineau-Desvoidy, 1830: 75. France (Paris).

Gonia trifaria Zeller, 1842: 841. Austria (Wien),

Poland (Reinerz and Szczecin).

Gonia maritima Perris, 1847: 495. France.

Distribution: PA: China, Egypt, Europe, Mongolia, Russia (W. Russia, W. Siberia), Transcaucasia.

Egyptian localities: Common throughout the country, particularly in: Coastal Strip: El-Burg, Mariout. Fayoum: ?. Eastern Desert: Wadi Rishrash, Wadi Wirak. Lower Nile Valley and Delta: Giza, Helwan, Kerdassa, Tanta. Sinai: El-Arish, Ein Gedeirat, Ein Moweileh, Gebel Moussa, Wadi El-Lega, Wadi Firan. Western Desert: Siwa Oasis. Upper Nile Valley: Asswan.

Dates of collection: February to October.

Gonia ornata Meigen, 1826

Gonia ornata Meigen, 1826: 3. Syntypes, males (MNHN). France (Lyon).

Gonia vittata Meigen, 1826: 3. Germany.

Gonia nervosa Meigen, 1826: 4. Germany (Hamburg).

Rhedia vernalis Robineau-Desvoidy, 1830: 74. France.

Rhedia diversa Robineau-Desvoidy, 1830: 77. France.

Gonia lateralis Zeller, 1842: 843. Poland (Glogow).

Distribution: PA: C. Asia (Kyrgyzstan, Tajikistan, Turkmenistan, Uzbekistan), China, Egypt, Europe (all), M. East, Mongolia, Russia (W. Russia, W. Siberia, S. Far East), Transcaucasia.

Egyptian localities: Common throughout the country, particularly in: Lower Nile Valley and Delta: Abu Rawash, Tanta. Upper Nile Valley: Qena.

Dates of collection: February to August.

Genus MENDELSSOHNIA Kugler

Mendelssohnia Kugler 1971: 69. Type species: Mendelssohnia sinaica Kugler, 1971, by original designation.

\section{Mendelssohnia sinaica Kugler, 1971}

Mendelssohnia sinaica Kugler, 1971: 70. Holotype male (TAU). Egypt (Sinai: Wadi El-Sheikh).

Distribution: Egypt, Israel.

Egyptian localities: Sinai: Wadi El Sheikh.

Dates of collection: Unknown.

Genus NEALSOMYIA Mesnil

Nealsomyia Mesnil, 1939: 31. Type species: Exorista (Alsomyia) triseriella Villeneuve, 1929, by original designation.

Nealsomyia triseriella (Villeneuve, 1929)

Exorista triseriella Villeneuve, 1929c: 185. Holotype male (CNC). Egypt (Wadi Hoff: nr. Helwan). 
Distribution: PA: Egypt, Israel.

Egyptian localities: Eastern Desert: Wadi Hoff.

Dates of collection: April to August.

Genus PSEUDOGONIA Brauer and Bergenstamm

Pseudogonia Brauer and Bergenstamm, 1889: 100.

Type species: Gonia cinerascens Rondani, 1859

(= Tachina rufifrons Wiedemann, 1830), by monotypy.

Gaediogonia Townsend, 1927: 71. Type species:

Gaediogonia jacobsoni Townsend, 1927 (= Tachina rufifrons Wiedemann, 1830), by original designation.

Pseudogonia fasciata (Wiedemann, 1819)

Gonia fasciata Wiedemann, 1819: 25. Syntypes, females (ZMUC). South Africa (Western Cape: Cape of Good Hope).

Rhedia capensis Robineau-Desvoidy, 1830: 77. South Africa (Western Cape: Cape of Good Hope).

Reaumuria lalandii Robineau-Desvoidy, 1830: 80. South Africa (Western Cape: Cape of Good Hope).

Distribution: AF: South Africa, Zimbabwe. PA: Andorra, Canary Is., Egypt, Portugal (including Azores, excluding Madeira), Spain.

Egyptian localities and dates of collection are not precisely known. Recorded from Egypt by Steyskal and El-Bialy 1967: 49).

\section{Pseudogonia rufifrons (Wiedemann, 1830)}

Tachina rufifrons Wiedemann, 1830: 318. Lectotype female (ZMUC). China.

Latreillia lalandii Robineau-Desvoidy, 1830: 106. South Africa (Western Cape: Cape of Good Hope).

Gonia cinerascens Rondani, 1859: 34. Italy (Parma).

Gonia munroi Curran, 1927b: 339. Tanzania.

Gonia ritchiei Cuthbertson and Munro, 1941: 109.

Nomen nudum.

Gonia munroi curran, 1927b: 339) Tanzania (Tanganyika) and South Africa.

Distribution: AF: Widespread, including Cape Verde, Nigeria, South Africa, Tanzania, U.A. Emirates, Yemen. AU: Australia, Hawaii, Melanesia, N. Australasian. OR: India, Indonesia, Malaysia, Myanmar, Orien. China [see O'Hara and Cerretti 2016: 19], Pakistan, Philippines, Ryukyu Is., Taiwan, Thailand. PA: C. Asia (Kyrgyzstan, Tajikistan, Turkmenistan, Uzbekistan), Egypt, all Europe (except British Is.), Japan, Kazakhstan, Korea, M. East (Israel), Mongolia, Pal. China [see O'Hara and Cerretti 2016: 19], Russia, Transcaucasia.

Egyptian localities: Coastal Strip: Mariout, Nuzha. Eastern Desert: Wadi Hoff. Gebel Elba: Wadi Edeib. Lower Nile Valley and Delta: Abu Rawash. Sinai: Wadi El-Tih.

Dates of collection: January to July.

Genus RAMONELLA Kugler

Ramona Kugler, 1980a: 40 (junior homonym of Ramona Casey, 1886). Type species: Ramona mesnili Kugler, 1980, by original designation.
Ramonella Kugler, 1980b: 67 (nomen novum for

Ramona Kugler, 1980).

Ramonella mesnili (Kugler, 1980)

Ramona mesnili Kugler, 1980a: 41. Holotype male (TAU). Israel (Negev: Ramon).

Distribution: AF: Yemen. PA: Canary Is., Egypt, Turkey, Israel.

Egyptian localities: Lower Nile Valley and Delta: ElMarg. Sinai: Wadi Taibe, Wadi Watir.

Dates of collection: May to September.

\section{Tribe WINTHEMIINI}

\section{Genus NEMORILLA Rondani}

Nemorilla Rondani, 1856: 66. Type species: Tachina maculosa Meigen, 1824, by original designation.

Nemorilla floralis (Fallén, 1810)

Tachina floralis Fallén, 1810: 287. Holotype male (NHRS). Sweden.

Tachina notabilis Meigen, 1824: 266. Sweden.

Tachina angustipennis Meigen, 1824: 333. W. Europe.

Tachina pabulina Meigen, 1824: 358. Germany

(Hamburg).

Exorista laticella Macquart, 1850: 373. France

(Lestrem).

Exorista arrogans Macquart, 1850: 374. France

(Lestrem).

Tachina intersita Walker, 1853: 72. England.

Nemorilla amica Rondani, 1859: 102. Italy (Parma).

Aubaea campestris Robineau-Desvoidy, 1863: 186.

France.

Pitthaea nebulosa Robineau-Desvoidy, 1863: 189.

France.

Distribution: PA: Egypt, Europe, Russia, Japan, North Korea.

Egyptian localities: Lower Nile Valley and Delta:

Bahtim, Ezbet El-Nakhl. Sinai: El-Arish.

Dates of collection: April to June.

\section{Subfamily PHASIINAE}

Tribe CYLINDROMYIINI

Genus BESSERIA Robineau-Desvoidy

Besseria Robineau-Desvoidy, 1830: 232. Type species: Besseria reflexa Robineau-Desvoidy, 1830, by monotypy.

Apostrophus Loew, 1871: 310, 311. Type species: Apostrophus suspectus Loew, 1871 (=Actia zonaria Loew, 1847), by subsequent designation of Coquillett (1910: 509).

Apostrophusia Townsend, 1933: 454. Type species: Apostrophus anthophilus Loew, 1871, by original designation.

Besseria anthophila (Loew, 1871)

Apostrophus anthophilus Loew, 1871: 310. Type(s), male(s), not located. Germany (Bavaria: MunichGeiselgasteig). 
Melia forcipata Bigot, 1881: 368. France (HautesAlpes: Queyras, Abries).

Distribution: NE: Alberta, Northwest Territories. PA: Egypt, Europe, Mongolia.

Egyptian localities: Coastal Strip: Dekhila, Mariout.

Dates of collection: June to August.

Genus CYLINDROMYIA Meigen

Cylindromyia Meigen, 1803: 279. Type species: Musca brassicaria Fabricius, 1775, by monotypy.

Ocyptera Latreille, 1804: 195. Type species: Musca brassicaria Fabricius, 1775, by subsequent designation of Curtis (1837: 629).

Exogaster Rondani, 1856: 78. Type species: Exogaster carinatus Rondani, 1856 (= Ocyptera rufifrons Loew, $1844)$, by original designation.

Ocypterula Rondani, 1856: 78. Type species: Ocyptera pusilla Meigen, 1824, by original designation.

Plesiocyptera Brauer and Bergenstamm, 1893: 56. Type species: Ocyptera bicolor Wiedemann, 1819, by monotypy.

Conopisoma Speiser, 1910: 144. Type species: Conopisoma miraculum Speiser, 1910, by original designation.

Formicocyptera Townsend, 1933: 451. Type species: Ocyptera atrata Fabricius, 1805, by original designation.

Cylindromyia intermedia (Meigen, 1824)

Ocyptera intermedia Meigen, 1824: 212. Holotype male (MNHN). ?Europe.

Ocyptera scalaris Loew, 1844: 240. Austria (Vienna).

Ocyptera excisa Loew, 1845: 171. Romania (Mehadia).

Ocyptera excisa var. rufiventris Strobl, 1906: 338. Spain

(Villa Rutis).

Ocyptera reinigi Enderlein, 1934: 132. Tajikistan

(Pamir: Karasu).

Distribution: Nearctic: Widespread. PA: C. Asia (Kyrgyzstan, Tajikistan, Turkmenistan, Uzbekistan), China, Egypt, Europe, Iran, Israel, Mongolia, Russia, Tadzhikistan, Transcaucasia, Ukraine, Uzbekistan.

Egyptian localities: Coastal Strip: Alexandria, Mariout, Max, Ramleh. Upper Nile Valley: Beni Suef.

Dates of collection: April to September.

Cylindromyia rufipes (Meigen, 1824)

Ocyptera rufipes Meigen, 1824: 215. Lectotype male (MNHN). France.

Distribution: AF: U.A. Emirates. OR: India, Pakistan. PA: Egypt, all Europe (except British Is., Scand.), Pakistan, Russia (W. Russia), Saudi Arabia, Transcaucasia, Ukraine.

Egyptian localities: Coastal Strip: Alexandria. Lower Nile Valley and Delta: Gebel Asfar, Kubba.

Dates of collection: October to February.
Dionomelia Kugler, 1978b: 346. Type species: Dionomelia hennigi Kugler, 1978, by original designation.

Dionomelia hennigi Kugler, 1978

Dionomelia hennigi Kugler, 1978b: 346. Holotype female (TAU). Israel (Dead Sea area: Ein Boqeq).

Distribution: AF: U.A. Emirates. PA: Andorra, Egypt, Israel, Portugal (including Azores, excluding Madeira), Spain.

Egyptian localities: Sinai.

Dates of collection: April to September.

Genus LEUCOSTOMA Meigen

Leucostoma Meigen, 1803: 279. Type species: Ocyptera simplex Fallén, 1815, by subsequent monotypy of Meigen (1824: 234).

\section{Leucostoma engeddense Kugler, 1966}

Leucostoma engeddense Kugler, 1966: 177. Holotype female (TAU). Israel (Dead Sea area: Ein Gedi).

Distribution: AF: South Africa, U.A. Emirates. PA: Algeria, Canary Is., Egypt, Israel, Madeira, NW Africa, Spain, Turkey.

Egyptian localities: Lower Nile Valley and Delta: Minia El-Qamh, Tanta.

Dates of collection: February to May.

Leucostoma obsidianum (Wiedemann, 1830)

Tachina obsidiana Wiedemann, 1830: 341. Lectotype female (SMF). Sudan (Nuba [as "Nubien"]).

Leucostoma marismortui Kugler, 1966: 179. Israel (Dead Sea area: Ein Gedi).

Distribution: AF: Sudan, Yemen. PA: Canary Is., Egypt, Europe, Israel, Malta, Saudi Arabia.

Egyptian localities: Upper Egypt: Asswan.

Dates of collection: Unknown.

\section{Tribe PHASIINI}

\section{Genus PHASIA Latreille}

Phasia Latreille, 1804: 195. Type species: Conops subcoleoptratus Linnaeus, 1767, by subsequent monotypy of Latreille (1805: 379).

Alophora Robineau-Desvoidy, 1830: 293. Type species: Syrphus hemipterus Fabricius, 1794, by subsequent designation of Robineau-Desvoidy (1863b: 226, as "Thereva hemiptera de Fabricius").

Hyalomya Robineau-Desvoidy, 1830: 298. Type species: Phasia semicinerea Meigen, 1824 (=Phasia pusilla Meigen, 1824), by subsequent designation of Westwood (1840: 140).

Phorantha Rondani, 1861b: 209. Type species: Phorantha musciformis Rondani, 1861 (= Conops subcoleoptratus Linnaeus, 1767), by monotypy.

Paralophora Girschner, 1887: 412 (as subgenus of Alophora Robineau-Desvoidy, 1830). Type species: Phasia pusilla Meigen, 1824, by monotypy.

Alophorella Townsend, 1912: 45. Type species: Thereva obesa Fabricius, 1798, by original designation. 
Phasia pusilla Meigen, 1824

Phasia pusilla Meigen, 1824: 198. Lectotype male (CNC). Germany (Stolberg).

Phasia semicinerea Meigen, 1824: 199. Germany (Stolberg).

Hyalomya carbonaria Robineau-Desvoidy, 1830: 300.

France (St. Sauveur).

Hyalomya corinna Robineau-Desvoidy, 1830: 301.

France.

Phasia vitripennis Zetterstedt, 1859: 6169. Sweden

(Skane).

Hyalomya chorea Robineau-Desvoidy, 1863: 266.

France.

Distribution: PA: C. Asia (Kyrgyzstan, Tajikistan, Turkmenistan, Uzbekistan), China, Egypt, Europe (all), Israel, Japan, Kazakhstan, Mongolia, Russia, Transcaucasia.

Egyptian localities and dates of collection are not precisely known.

\section{Subfamily TACHININAE \\ Tribe ERNESTIINI}

\section{Genus EURITHIA Robineau-Desvoidy}

Erigone Robineau-Desvoidy, 1830: 65 (junior homonym of Erigone Audouin, 1826). Type species: Erigone anthophila Robineau-Desvoidy, 1830, by subsequent designation of Townsend (1932: 42).

Eurithia Robineau-Desvoidy, 1844: 24. Type species: Erigone puparum Robineau-Desvoidy, 1830 (= Tachina caesia Fallén, 1810), by monotypy.

\section{Eurithia castellana (Strobl, 1906)}

Erigone castellana Strobl, 1906: 338. Holotype male (NMBA). Spain (Madrid). Holotype may be lost, see O'Hara et al. (2009).

Distribution: PA: Albania, Bosnia and Herzegovina, Bulgaria, China, Croatia, Egypt, Greece, Israel, Montenegro, Macedonia, Serbia, Slovenia, Transcaucasia.

Egyptian localities: Coastal Strip: El-Burg, Mariout. Eastern Desert: Ismailia. Fayoum: ?. Lower Nile Valley and Delta: Abu Rawash. Upper Nile Valley: Asswan.

Dates of collection: January to October.

Genus LINNAEMYA Robineau-Desvoidy

Linnaemya Robineau-Desvoidy, 1830: 52. Type species: Linnaemya silvestris Robineau-Desvoidy, 1830 (= Tachina vulpina Fallén, 1810), by subsequent designation of Robineau-Desvoidy (1863a: 131).

Bonellia Robineau-Desvoidy, 1830: 56 (junior homonym of Bonellia Rolando, 1822). Type species: Bonellia tessellans Robineau-Desvoidy, 1830, by subsequent designation of Townsend (1916: 6).

Elachipalpus Rondani, 1850: 169. Type species: Micropalpus longirostris Macquart, 1845 (junior primary homonym of Micropalpus longirostris Macquart, 1844; = Elachipalpus rondanii Townsend, 1916), by original designation.
Tachinomima Brauer and Bergenstamm, 1891: 383.

Type species: Tachinomima expetens Brauer and Bergenstamm, 1891 (= Micropalpus longirostris Macquart, 1844), by monotypy.

Hecatoepalpus Townsend, 1933: 467. Type species: Micropalpus prohecate Speiser, 1910, by original designation.

Micropalpinus Enderlein, 1937: 441. Type species: Micropalpus pallidus Jaennicke, 1867, by original designation.

Gymmantia Enderlein, 1937: 441. Type species: Micropalpus alboscutellatus Speiser, 1910, by original designation.

Linnaemya comta (Fallén, 1810)

Tachina comta Fallén, 1810: 277. Unspecified type(s), females (NHRS) Sweden.

Tachina compta Meigen, 1824: 262. Unjustified emendation of comta Fallén.

Tachina fulgens Meigen, 1824: 259. Austria, France and Germany.

Linnaemya heraclei Robineau-Desvoidy, 1830: 53. France (Paris).

Linnaemya analis Robineau-Desvoidy, 1830: 54. France (Angers).

Linnaemya aestivalis Robineau-Desvoidy, 1830: 54. France.

Linnaemya borealis Robineau-Desvoidy, 1830: 54. France.

Bonnetia longipes Robineau-Desvoidy, 1830: 55.

France.

Bonnetia oenanthis Robineau-Desvoidy, 1830: 56. France (Paris).

Micropalpus humeralis Robineau-Desvoidy, 1863: 119. New name for Bonnetia longipes Robineau-Desvoidy, 1830.

Distribution: AF: Sudan. NE: Widespread. OR: India, Nepal. PA: C. Asia (Kyrgyzstan, Tajikistan, Turkmenistan, Uzbekistan), China, Egypt, Europe (all), Kazakhstan, Israel, Mongolia, Russia (W. Russia, W. Siberia, E. Siberia, S. Far East), Taiwan, Transcaucasia.

Egyptian localities: Eastern Desert: Wadi Garawi, Wadi Rishrash. Gebel Elba: ?. Lower Nile Valley and Delta: Mansouriya.

Dates of collection: January to March.

\section{Linnaemya latigena Kugler, 1977}

Linnaemyia latigena Kugler, 1977: 3. Holotype male (TAU). Egypt (Sinai: Bir Gifgafa [as "Refidim"]).

Distribution: AF: U.A. Emirates. PA: Egypt, Israel.

Egyptian localities: Sinai: Bir Gifgafa, Mitla, Wadi Firan.

Dates of collection: April.

\section{Linnaemya petiolata Kugler, 1971}

Linnaemya petiolata Kugler, 1971: 80. Unspecified type(s), male(s) (TAU). Egypt (South Sinai: Abu Marawath). 
Distribution: PA: Egypt.

Egyptian localities: Sinai: Abu Marawath.

Dates of collection: April to August.

Linnaemya soror Zimin, 1954

Linnaemyia soror Zimin, 1954: 266. Holotype male (ZIN). Tajikistan (Gorno-Badakhshan: Khorugh).

Distribution: PA: Canary Is., C. Asia (Kyrgyzstan, Tajikistan, Turkmenistan, Uzbekistan), China, Egypt, W. Europe (Andorra, Portugal (including Azores, excluding Madeira), Spain), S. Europe (Albania, Bosnia and Herzegovina, Bulgaria, Croatia, Greece, Montenegro, Macedonia, Serbia, Slovenia), Israel, North Africa, Russia, Transcaucasia, Turkmenia, Uzbekistan. OR: India, Nepal.

Egyptian localities: Pyramids.

Dates of collection: September.

\section{Tribe GRAPHOGASTRINI}

\section{Genus PHYTOMYPTERA Rondani}

Phytomyptera Rondani, 1845: 32, 33. Type species: Phytomyptera nitidiventris Rondani, 1845 (= Tachina nigrina Meigen, 1824), by monotypy.

Elfia Robineau-Desvoidy, 1850: 190. Type species: Actia cingulata Robineau-Desvoidy, 1830, by subsequent designation of Robineau-Desvoidy (1863a: 672).

Phytomyptera lacteipennis Villeneuve, 1934

Phytomyptera lacteipennis Villeneuve, 1934b: 71. Lectotype female (CNC). Egypt (Suez).

Distribution: AF: U.A. Emirates. PA: Egypt, Europe, Israel, Libya, Mongolia, Russia (W. Russia).

Egyptian localities: Eastern Desert: Suez Road.

Dates of collection: March.

\section{Tribe MINTHOINI}

\section{Genus MINTHO Robineau-Desvoidy}

Mintho Robineau-Desvoidy, 1830: 216. Type species: Musca compressa Fabricius, 1787, by subsequent designation of Rondani (1856: 79).

Mintho compressa (Fabricius, 1787)

Musca compressa Fabricius, 1787: 346. Unspecified type(s) (ZMUC). Spain.

Mintho capensis Robineau-Desvoidy, 1830: 217. South Africa (Western Cape: Cape of Good Hope).

Tachina isis Wiedemann, 1830: 304. Egypt.

Ocyptera vittata Costa, 1844: 94. Italy (Cervinara and Guagnano).

Sericocera algira Macquart, 1849: 480. Algeria.

Dexia thala Walker, 1849: 845. Morocco (Tangier).

Dexia isapis Walker, 1849: 848. Egypt.

Distribution: AF: Widespread, including D.R. Congo, Eritrea, Kenya, Nigeria, Somalia, South Africa, Sudan, Tanzania, Yemen. PA: Algeria, Canary Is., Egypt, Europe, Israel, Libya, Morocco, Transcaucasia, Tunisia.
Egyptian localities. Coastal Strip: Alexandria, Amrya. Lower Nile Valley and Delta: Abu Rawash, Ain Shams, Beni Meggon, Cairo, Dokki, El-Baragil, Ezbet El-Nakhl, Giza, Kafr Hakim, Kerdassa, Mansouriya, Maadi, Nahya, Shubra, Sinai: El-Arish. Sinai: El-Arish. Upper Nile Valley: Assiout, Asswan.

Dates of collection. Throughout the year.

\section{Genus PLESINA Meigen}

Plesina Meigen, 1838: 214. Type species: Tachina phalerata Meigen, 1824, by monotypy.

Xanthopetia Townsend, 1933: 452. Type species: Tachina fascipennis Wiedemann, 1830, by original designation.

Kugleria Verbeke, 1970: 299 (junior homonym of Kugleria Bouwman, 1938). Type species: Plesina fascipennis claripennis Mesnil, by monotypy.

\section{Plesina claripennis Mesnil, 1953}

Plesina claripennis Mesnil, 1953b: 166. Holotype (not located). Israel (Jerusalem).

Distribution: Egypt, Israel.

Egyptian localities: Sinai.

Dates of collection: ?

\section{Plesina deserticola Kugler, 1978}

Plesina deserticola Kugler, 1978a: 93. Holotype male (TAU). Egypt (Sinai: Wadi Shag).

Distribution: PA: Egypt, Israel.

Egyptian localities: Sinai: Wadi Shag (South Sinai).

Dates of collection: ?

\section{Genus ROSSIMYIOPS Mesnil}

Rossimyiops Mesnil, 1953a: 145. Type species: Rossimyiops whiteheadi Mesnil, 1953, by monotypy.

Mesnilomyia Kugler, 1972: 103. Type species: Mesnilomyia magnifica Kugler, 1972, by original designation.

Persedea Richter, 2001: 25. Type species: Persedea exquisita Richter, 2001, by original designation.

Rossimyiops achilleae (Kugler, 1972)

Mesnilomyia achilleae Kugler, 1972: 107. Holotype male (TAU). Israel ('Arad).

Distribution: PA: Egypt, Israel.

Egyptian localities: Sinai: St. Catherine, Wadi ElArbaein, Wadi Shag.

Dates of collection: July.

Rossimyiops magnificus (Kugler, 1972)

Mesnilomyia magnifica Kugler, 1972: 105. Holotype male (TAU). Israel ('Arad).

Distribution: PA: Egypt, Iraq, Israel.

Egyptian localities: Sinai: Tarfat El-Kadarin, Wadi Nazeb, Wadi Sa'al, Nueiba'.

Dates of collection: April to September.

\section{Tribe SIPHONINI}

Genus ACTIA Robineau-Desvoidy 
Actia Robineau-Desvoidy, 1830: 85. Type species: Roeselia lamia Meigen, 1838, by designation under the plenary powers of ICZN.

Gymnophthalma Lioy, 1864: 1341. Type species: Tachina crassicornis Meigen, 1824, by monotypy.

Gymnopareia Brauer and Bergenstamm, 1889: 103. Type species: Tachina crassicornis Meigen, 1824, by monotypy.

\section{Actia crassicornis (Meigen, 1824)}

Tachina crassicornis Meigen, 1824: 351. Syntypes, males and females (MNHN). Probably Germany (Stolberg). See O'Hara (2009).

Thryptocera bicolor Macquart, 1834: 312. France (Lille).

Thryptocera flavipalpis Macquart, 1848: 135. Switzerland (Ütliberg).

Thryptocera nigripalpis Robineau-Desvoidy, 1851: 182. France.

Thryptocera palpalis Rondani, 1859: 14. Italy (Parma).

Thryptocera claripennis Robineau-Desvoidy, 1863: 716 France,

Thryptocera scutellaris Rondani, 1865: 195. Italy (Parma).

Distribution: PA: China, Egypt, Europe (all), Japan, Kazakhstan, Mongolia, Russia, Transcaucasia.

Egyptian localities: Coastal Strip: Alexandria. Eastern Desert: Ismailia. Lower Nile Valley and Delta: Abu Rawash, Dokki, Giza, Kerdassa. Sinai: El-Arish. Upper Nile Valley: Assiout.

Dates of collection: April to September.

Genus PERIBAEA Robineau-Desvoidy

Herbstia Robineau-Desvoidy, 1851: 184 (junior homonym of Herbstia Edwards, 1834). Type species: Herbstia tibialis Robineau-Desvoidy, 1851, by monotypy.

Peribaea Robineau-Desvoidy, 1863a: 720. Type species: Peribaea apicalis Robineau-Desvoidy, 1863 (= Herbstia tibialis Robineau-Desvoidy, 1851), by subsequent designation of Coquillett (1910: 587).

Strobliomyia Townsend, 1926a: 31. Type species: Tryptocera fissicornis Strobl, 1910 (as "Thryptocera fissicornis") (= Thryptocera setinervis Thomson, 1869), by original designation.

\section{Peribaea orbata (Wiedemann, 1830)}

Tachina orbata Wiedemann, 1830: 336. Neotype female (BMNH). India (Assam: Azra).

Gymnopareia (Actia) aegyptia Villeneuve, 1913a: 508. Egypt (Qaliûb).

Actia nigripes Curran, 1927a: 6. D.R. Congo (BasCongo: Boma).

Strobliomyia sororcula Mesnil, 1954: 16. D.R. Congo (Nord-Kivu: Rutshuru).

Distribution: AF: Widespread. OR: India, Indonesia, Orien. China [see O'Hara and Cerretti 2016: 19], Malaysia, Myanmar, Philippines, Ryukyu Is., Sri Lanka,
Taiwan, Thailand. PA: Japan, Israel, North Africa, P. China. AU: Australia, Melanesia, Micronesia, N. Australasian.

Egyptian localities: Lower Nile Valley and Delta: Giza, Sidi Ghazi, Torah. Upper Nile Valley: Beni Morr (Assiout).

Dates of collection: May to August.

\section{Peribaea palaestina (Villeneuve, 1934)}

Actia palaestina Villeneuve, 1934a: 57. Holotype female (SMNS). Israel, (Rehovot [as "Rehoboth”]).

Actia alipes Villeneuve, 1942: 134. Egypt (Asswan [as "Assuan"]).

Distribution: AF: U.A. E., Yemen. PA: C. Asia (Kyrgyzstan, Tajikistan, Turkmenistan, Uzbekistan), China, Egypt, Iran, Israel.

Egyptian localities and dates of collection are not precisely known.

\section{Genus SIPHONA Meigen}

Crocuta Meigen, 1800: 39. Name suppressed by ICZN.

Siphona Meigen, 1803: 281. Type species: Musca geniculata De Geer, 1776, by designation under the plenary powers of ICZN.

Subgenus SIPHONA Meigen, 1803 -Nominotypical subgenus.

Siphona (Siphona) efflatouni Mesnil, 1960

Siphona efflatouni Mesnil, 1960: 188. Holotype female (CNC). Egypt (Mariout).

Distribution: PA: Egypt, Israel.

Egyptian localities: Coastal Strip: Mariout. Sinai: El-Arish. Dates of collection: March to August.

\section{Tribe TACHININI}

\section{Genus PELETERIA Robineau-Desvoidy}

Peleteria Robineau-Desvoidy, 1830: 39. Type species: Peleteria abdominalis Robineau-Desvoidy, 1830, by subsequent designation of Coquillett (1910: 586).

Cuphocera Macquart, 1845: 267. Type species: Micropalpus ruficornis Macquart, 1835, by original designation.

Acuphocera Townsend, 1926b: 37. Type species: Acuphocera sumatrensis Townsend, 1926 (= Tachina iavana Wiedemann, 1819), by original designation.

Pleropeleteria Villeneuve, 1916: 470. Type species: Dejeania (Pleropeleteria) peringueyi Villeneuve, 1916 (= Tachina lithanthrax Wiedemann, 1830), by monotypy.

Peletieriana Mesnil, 1970a: 951 (as subgenus of Peleteria Robineau-Desvoidy, 1830, as "Peletieria"). Type species: Echinomyia rustica Karsch, 1886, by original designation.

Peleteria meridionalis (Robineau-Desvoidy, 1830)

Faurella meridionalis Robineau-Desvoidy, 1830: 41. Type(s) not located. France.

Echinomyia ruficeps Macquart, 1845: 263. Italy.

Peleteria vernalis Robineau-Desvoidy, 1863: 617. France (Hyeres). 
Peleteria pedemontana Robineau-Desvoidy, 1863: 619. Italy.

Peleteria algira Robineau-Desvoidy, 1863: 621. Algeria.

Peletieria capitata Zimin, 1961: 265. Turkmenistan

(Chandyr Valley).

Distribution: PA: Algeria, Egypt, Isael, Italy, Kirghizia, Russia, Tadzhikistan, Transcaucasia, Uzbekistan.

Egyptian localities: Coastal Strip: Alexandria, Mariout. Fayoum: ?. Lower Nile Valley and Delta: Kerdassa, Dokki, Maadi. Sinai: El-Arish. Upper Nile Valley: Assiout.

Dates of collection: November to April.

List of doubtful taxa (Nomina Dubia) described from Egypt: (see Herting and Dely-Draskovits 1993)

- Catabucentes abdominalis Villeneuve, 1934b: 72. Egypt (Lower Egypt).

- Eurigaster incisus Walker, 1871: 340. Egypt (Hot Tamanib).

- Eurigaster leucomelas Walker, 1871: 339. Egypt.

- Frontina latifascia Walker, 1871: 340. Egypt (Wadi Genneh).

- Reaumuria olivieri Robineau-Desvoidy, 1830: 82. Egypt.

- Reaumuria virescens Robineau-Desvoidy, 1863b: 741. Egypt.

\section{Conclusion}

As a final conclusion, this catalogue treats a total of 72 tachinid species belonging to 42 genera, 15 tribes and 4 subfamilies in Egypt. Synonymies, type localities, type depositories, world distributions by biogeographic realm(s) and country, Egyptian localities and dates of collection are provided for all Egyptian taxa.

\section{Appendix}

Checklist of the Tachinidae of Egypt

Subfamily DEXIINAE

Tribe DEXIINI

Genus TRIXICEPS Villeneuve

- Trixiceps magnipalpis (Bezzi, 1922)

\section{Tribe VORIINI}

Genus HYPOVORIA Villeneuve

- Hypovoria hilaris (Villeneuve, 1913)

Genus NANOPLAGIA Villeneuve

- Nanoplagia sinaica (Villeneuve, 1909)

Genus PERISCEPSIA Gistel

Subgenus PERISCEPSIA Gistel

- Periscepsia (Periscepsia) handlirschi (Brauer and von Bergenstamm, 1891)
Genus UCLESIA Girschner

- Uclesia petiolata (Villeneuve, 1929)

Genus WAGNERIA Robineau-Desvoidy

- Wagneria cunctans (Meigen, 1824)

Subfamily EXORISTINAE

Tribe ERYCIINI

Genus AMELIBAEA Mesnil

- Amelibaea ?signifera (Villeneuve, 1929)

Genus APLOMYA Robineau-Desvoidy

- Aplomya confinis (Fallén, 1820)

- Aplomya metallica (Wiedemann, 1824)

Genus CADURCIELLA Villeneuve

- Cadurciella tritaeniata (Rondani, 1859)

Genus CESTONIA Rondani

- Cestonia cineraria Rondani, 1861

- Cestonia rutilans Villeneuve, 1929

Genus CESTONIONERVA Villeneuve

- Cestonionerva petiolata (Villeneuve, 1910)

Genus DRINO Robineau-Desvoidy

Subgenus PALEXORISTA Townsend

- Drino (Palexorista) gilva (Hartig, 1838)

- Drino (Palexorista) imberbis (Wiedemann, 1830)

- Drino (Palexorista) inconspicua (Meigen, 1830)

Subgenus ZYGOBOTHRIA Mik

- Drino (Zygobothria) atropivora (Robineau-Desvoidy, 1830)

Genus THECOCARCELIA Townsend

- Thecocarcelia acutangulata (Macquart, 1851)

Tribe ETHILLINI

Genus ATYLOMYIA Brauer

- Atylomyia albifrons Villeneuve, 1911

Tribe EXORISTINI

Genus ALLOPROSOPAEA Villeneuve 
- Alloprosopaea efflatouni Villeneuve, 1923

Genus CHAETORIA Becker

- Chaetoria stylata Becker, 1908

Genus CHETOGENA Rondani

- Chetogena aegyptiaca (Villeneuve, 1923)

- Chetogena cercosa (Kugler, 1980)

- Chetogena sinaica (Villeneuve, 1909)

Genus EXORISTA Meigen.

Subgenus ADENIA Robineau-Desvoidy

- Exorista (Adenia) rustica (Fallén, 1810)

Subgenus EXORISTA Meigen

- Exorista (Exorista) fasciata (Fallén, 1820)

- Exorista (Exorista) larvarum (Linnaeus, 1758)

Subgenus PODOTACHINA Brauer and Bergenstamm

- Exorista (Podotachina) sorbillans (Wiedemann, 1830)

Subgenus PTILOTACHINA Brauer and Bergenstamm

- Exorista (Ptilotachina) rutilans Mesnil, 1970

- Exorista (Ptilotachina) xanthaspis (Wiedemann, 1830)

Genus MACULOSALIA Mesnil

- Maculosalia maculosa (Villeneuve, 1909)

\section{Tribe GONIINI}

Genus BLEPHARELLA Macquart

- Blepharella versatilis (Villeneuve, 1910)

Genus CLEMELIS Robineau-Desvoidy

- Clemelis apicalis Villeneuve, 1923

Genus GONIA Meigen

- Gonia atra Meigen, 1826

- Gonia bimaculata Wiedemann, 1819

- Gonia capitata (De Geer, 1776)

- Gonia ornata Meigen, 1826

Genus MENDELSSOHNIA Kugler

- Mendelssohnia sinaica Kugler, 1971
Genus NEALSOMYIA Mesnil

- Nealsomyia triseriella (Villeneuve, 1929)

Genus PSEUDOGONIA Brauer and Bergenstamm

- Pseudogonia fasciata (Wiedemann, 1819)

- Pseudogonia rufifrons (Wiedemann, 1830)

Genus RAMONELLA Kugler

- Ramonella mesnili (Kugler, 1980)

Tribe WINTHEMIINI

Genus NEMORILLA Rondani

- Nemorilla floralis (Fallén, 1810)

\section{Subfamily PHASIINAE}

Tribe CYLINDROMYIINI

Genus BESSERIA Robineau-Desvoidy

- Besseria anthophila (Loew, 1871)

Genus CYLINDROMYIA Meigen

- Cylindromyia intermedia (Meigen, 1824)

- Cylindromyia rufipes (Meigen, 1824)

Tribe LEUCOSTOMATINI

Genus DIONOMELIA Kugler

- Dionomelia hennigi Kugler, 1978

Genus LEUCOSTOMA Meigen

- Leucostoma engeddense Kugler, 1966

- Leucostoma obsidianum (Wiedemann, 1830)

\section{Tribe PHASIINI}

Genus PHASIA Latreille

- Phasia pusilla Meigen, 1824

Subfamily TACHININAE

Tribe ERNESTIINI

Genus EURITHIA Robineau-Desvoidy

- Eurithia castellana (Strobl, 1906)

Genus LINNAEMYA Robineau-Desvoidy

- Linnaemya comta (Fallén, 1810)

- Linnaemya latigena Kugler, 1977 
- Linnaemya petiolata Kugler, 1971

- Linnaemya soror Zimin, 1954

Tribe GRAPHOGASTRINI Genus PHYTOMYPTERA Rondani

- Phytomyptera lacteipennis Villeneuve, 1934

\section{Tribe MINTHOINI}

Genus MINTHO Robineau-Desvoidy

- Mintho compressa (Fabricius, 1787)

Genus PLESINA Meigen

- Plesina claripennis Mesnil, 1953

- Plesina deserticola Kugler, 1978

Genus ROSSIMYIOPS Mesnil

- Rossimyiops achilleae (Kugler, 1972)

- Rossimyiops magnificus (Kugler, 1972)

\section{Tribe SIPHONINI}

Genus ACTIA Robineau-Desvoidy

- Actia crassicornis (Meigen, 1824)

Genus PERIBAEA Robineau-Desvoidy

- Peribaea orbata (Wiedemann, 1830)

- Peribaea palaestina (Villeneuve, 1934)

Genus SIPHONA Meigen

Subgenus SIPHONA Meigen, 1803

- Siphona (Siphona) efflatouni Mesnil, 1960

Tribe TACHININI.

Genus PELETERIA Robineau-Desvoidy.

Peleteria meridionalis (Robineau-Desvoidy, 1830).

\section{Abbreviations}

List of abbreviations used throughout the catalogue

C.: Central; E.: East; Eur.: Europe; ICZN: International Commission on Zoological Nomenclature; I., Is.: Island, islands; M.: Middle; N.: North; Ne.: Northeast; Nw.: Northwest; nr.: near; Pal.: Palaearctic; S.: South; St.: Saint; var.: Variety; W.: West

\section{Museum abbreviations used throughout the catalogue}

BMNH: Natural History Museum [formerly British Museum (Natural History)], London, United Kingdom; CNC: Canadian National Collection of Insects,

Agriculture and Agri-Food Canada, Ottawa, Ontario, Canada; EFC: Collection of Entomology Department, Faculty of Science, Cairo University, Egypt (Efflatoun's collection); LSUK: Linnaean Collections, The Linnean Society of
London, London, United Kingdom; MHNL: Musée d'Histoire Naturelle de Lille, Lille, France; MNHN: Muséum National d'Histoire Naturelle, Paris, France; MRSN: Museo Regionale di Scienze Naturali, Torino [Turin], Italy; MZF: Museo Zoologico "La Specola", Firenze [Florence], Italy; MZLU: Museum of Zoology, Lund University, Lund, Sweden; NHMW: Naturhistorisches Museum Wien, Wien [Vienna], Austria; NHRS: Naturhistoriska riksmuseet [Swedish Museum of Natural History], Stockholm, Sweden; NMBA: Naturhistorisches Museum der Benediktiner-Abtei Admont, Admont, Austria; PPDD: Ministry of Agriculture Collection, Plant Protection Research Institute, Dokki, Giza, Egypt; SMF: Forschungsinstitut und Naturmuseum Senckenberg, Frankfurt am Main, Germany; SMNS: Staatliches Museum für Naturkunde, Stuttgart, Germany; TAU: Tel Aviv University, Tel Aviv, Israel; USNM: National Museum of Natural History [formerly United States National Museum], Smithsonian Institution, Washington, District of Columbia, USA; ZIN: Zoological Institute, Russian Academy of Sciences, St. Petersburg, Russia; ZMHB: Museum für Naturkunde der Humboldt-Universität zu Berlin, Berlin, Germany; ZMUC: Zoological Museum, Natural History Museum of Denmark, University of Copenhagen, Copenhagen, Denmark; ZMUH: Universität von Hamburg, Zoologisches Institut und Zoologisches Museum, Hamburg, Germany

Biogeographic Realm abbreviations used throughout the catalogue AF: Afrotropical; AU: Australasian; NE: Nearctic; NT: Neotropical; OR: Oriental; PA: Palaearctic

\section{Acknowledgements}

I would like to thank Dr. Amnon Freidberg and Dr. Tatyana Novoselsky, Tel Aviv University, Israel for supplying me with some relevant papers. I am grateful to Dr. Ashraf Al-Torky, Plant Protection Institute, Ministry of Agriculture, Egypt, for his help throughout this study.

Funding

No funding.

Availability of data and materials

Data supporting the conclusions of this article are presented in the main manuscript.

\section{Author's contributions}

This is a single author study. The author read and approved the final manuscript.

Ethics approval and consent to participate

Not applicable.

Consent for publication

Not applicable.

\section{Competing interests}

The author declares that he has no competing interests.

\section{Publisher's Note}

Springer Nature remains neutral with regard to jurisdictional claims in published maps and institutional affiliations.

Received: 18 December 2017 Accepted: 19 March 2018

Published online: 30 May 2018

\section{References}

Becker T (1908) Dipteren der Kanarischen Inseln. Mitt Zool Mus Berlin 4:1-180

Becker T (1910) Dipteren aus Südarabien und von der Insel Sokótra. Kaiserl Akad Wiss Wien, Math Naturwiss Kl, Denkschr 71:131-160

Bezzi M (1922) Contributo allo studio della fauna Libica. Ditteri di Cirenaica raccolti dal Rev Miss Don Vito Zanon Mem Soc entomol ital 1: 140-157

Bezzi M, Lamb CG (1926) Diptera (excluding Nematocera) from the island of Rodriguez. Trans Entomol Soc Lond 58(1925):537-573

Bigot JMF (1881) Diptères nouveaux ou peu connus. 17e partie. XXVI. Ann Soc Entomol Fr, Sér. 6 1:363-371

Bigot JMF (1888) Enumeration des Dipteres recueillis en Tunisie dans la Mission de 1884 par M. Valery Mayet membre de la Mission de l'Exploration Scientifique de la Tunisie, et description des speces nouvelles. Exploration 
scientifique de la Tunisie, publiée sous les auspices du Ministère de I'Instruction Publique. Zoologie, p 11

Brauer F (1893) Vorarbeiten zu einer Monographie der Muscaria schizometopa (exclusive Anthomyidae) von Prof. Dr. Fr. Brauer und Julius Edl. v. Bergenstamm. Verh k k zool-bot Ges Wien 43(Abhandlungen):447-525

Brauer F (1898) Beiträge zur Kenntniss der Muscaria schizometopa. I. Bemerkungen zu den Originalexemplaren der von Bigot, Macquart und Robineau-Desvoidy beschriebenen Muscaria schizometopa aus Sammlung des Herrn G.H. Verrall. Zweite Folge. Sber Akad Wiss Wien, Abteilung I 107:493-546

Brauer F, von Bergenstamm JE (1889) Die Zweiflügler des Kaiserlichen museums Zu Wien. IV. Vorarbeiten zu einer Monographie der Muscaria schizometopa (exclusive Anthomyidae). Pars I. Denkschr Akad Wiss Wien 56(1):69-180 + 11 pls

Brauer F, von Bergenstamm JE (1891) Die Zweiflügler des Kaiserlichen Museums zu Wien. V. Vorarbeiten zu einer Monographie der Muscaria schizometopa (exclusive Anthomyidae). Pars II. F Tempsky, Wien:142

Brauer F, von Bergenstamm JE (1893) Die Zweiflügler des Kaiserlichen Museums zu Wien. VI. Vorarbeiten zu einer Monographie der Muscaria Schizometopa (exclusive Anthomyidae). Pars III. F Tempsky, Wien:152

Cerretti P, Freidberg A (2009) Updated checklist of the Tachinidae of Israel. Tachinid Times 22:9-16

Cerretti P, Tschorsnig HP (2010) Annotated host catalogue for the Tachinidae (Diptera) of Italy. Stutt Beitr Naturk, Serie A (Biologie) 3:305-340

Coquillett DW (1910) The type-species of the North American genera of Diptera. Proc U S Natl Mus 37(1719):499-647

Costa OG (1844) Descrizione di dodici specie nuove dell'ordine de'Ditteri ed illustrazione di altre quattordici meno ovvie raccolte nella state del 1834. Atti Accad Sci Napoli 5(2):81-107

Curran CH (1927a) New African Tachinidae. Am Mus Novit 258:1-20

Curran CH (1927b) Studies in African Tachinidae (Diptera). Bull Entomol Res 17: 319-340

Curran CH (1927c) Studies in African Tachinidae (Diptera). Bull Entomol Res 18: 103-128. https://doi.org/10.1017/S0007485300019787

Curtis J (1837) British Entomology; being illustrations and descriptions of the genera of insects found in Great Britain and Ireland: containing coloured figures from nature of the most rare and beautiful species, and in many instances of the plants upon which they are found, vol 14. Privately published, London, pp 626-673

Cuthbertson A, Munro HK (1941) Some records of tachinid parasites and their insect hosts in southern Africa. Trans Rhodesia Sci Assoc 38:88-118

De Geer C (1776) Memoires pour servir a l'histoire des insectes. Tome sixieme. P. Hesselberg, Stockholm, p viii +522

El-Hawagry M (2015) Catalogue of Superfamily Asiloidea. Lap Lambert Academic Publishing, Saarbrücken, p 196

El-Hawagry M (2017) Catalogue of Egyptian Tephritoidea (Diptera: Schizophora: Acalyptratae). Zootaxa 4299(2):151-190. https://doi.org/10.11646/zootaxa.4299.2.1

El-Hawagry M, Gilbert F (2014) Zoogeographical affinities and faunal relationships of bee flies (Diptera: Bombyliidae) in Egypt. Zool Middle East 60(1):50-56. https://doi.org/10.1080/09397140.2014.892339

Enderlein G (1934) Entomologische Ergebnisse der Deutsch-Russischen alaiPamir-expedition 1928 (III). 1. Diptera. Dtsch Entomol Z 1933:129-146

Enderlein G (1937) Dipterologica. IV. Sitz. Ges Nat Freunde Berlin 1936(3):431-443

Fabricius JC (1787) Mantissa insectorum sistens species nuper detectas adiectis synonymis, observationibus, descriptionibus, emendationibus, vol 2, Hafniae, Impensis C. G. Proft (= Copenhagen, Denmark), p 382

Fallén CF (1810) Försök att bestämma de i Sverige funne flugarter, som kunna föras till slägtet Tachina. K Svenska Vetensk Akad Handl, Ser. 2 31:253-287

Fallén CF (1820a) Monographia Muscidum Sveciae. [part I.]. Berlingianis, Lundae [= Lund], pp 1-12

Fallén CF (1820b) Monographia Muscidum Sveciae. [part III.]. Berlingianis, Lundae [= Lund], pp 25-40

Girschner E (1887) Die europäischen Arten der Dipterengattung Alophora. Zeitsch Ges Naturwiss 60:375-426

Girschner E (1901) Ueber eine neue Tachinide und die Scutellarbeborstung der Musciden. Wien Entomol Zeit 20:69-72

Gistel J (1848) Naturgeschichte des Thierreichs. Für höhere Schulen. R. Hoffmann, Stuttgart, $\mathrm{p} x \mathrm{xi}+216$

Hartig T (1838) Über die parasitischen Zweiflügler des Waldes. Jber Fortschr forsiwiss Forst Naturk 1:275-306

Hermann F, Villeneuve J (1909) Diptera. Aus der Sinaihalbinsel. In: Kneucker A (ed) Zoologische Ergebnisse zweier in den Jahren 1902 und 1904 durch die Sinaihalbinsel unternommener botanischer Studienreisen nebst zoologischen
Beobachtungen aus Ägypten, Palästina und Syrien, pp 147-159 Verh. naturw. Ver. Karlsruhe, 21: 79-165

Herting B (1984) Catalogue of Palearctic Tachinidae (Diptera). Stuttg Beitr Naturk, Serie A (Biologie) 369:1-228

Herting B, Dely-Draskovits Á (1993) Family Tachinidae, pp. 118-458. In: Soós Á, Papp L (eds) Catalogue of Palaearctic Diptera. Volume 13. Anthomyiidae-Tachinidae. Hungarian Natural History Museum, Budapest, p 624

ICZN (1963) International Commission on Zoological Nomenclature. Opinion 678 The suppression under the Plenary Powers of the pamphlet published by Meigen, 1800. Bull Zool Nom 20: 339-342.

ICZN (1999) International code of zoological nomenclature (4 ed.). International Trust for Zoological Nomenclature, London. http://www.nhm.ac.uk/hostedsites/iczn/code/.

Irwin ME, Schlinger El, Thompson FC (2003) Diptera, true flies. In: Goodman SM, Benstead JP (eds) The natural history of Madagascar. The University of Chicago Press, Chicago, pp 692-698

Kugler J (1966) Species of the genus Leucostoma (Tachinidae, Phasiinae) in Israel. Isr J Zool 15:173-182

Kugler J (1971) Tachinidae of Israel. IV. Description of ten new species. Isr J Zool 20:69-88

Kugler J (1972) Tachinidae of Israel, V. Mesnilomyia and Palmonia, two new genera of Tachinidae (Diptera). Isr J Zool 21:103-112

Kugler J (1977) Neue Tachinidae aus Israel (Diptera). Stuttg Beitr Naturk, Serie A (Biologie) 301:1-14

Kugler J (1978a) A revision of the tachinid fly genus Plesina (Diptera: Tachinidae). Entomol Ger 4:84-96

Kugler J (1978b) Leucostoma edentata n. sp. and Dionomelia hennigi n. gen., n. sp. , two new Leucostomatini from Israel (Diptera: Tachinidae: Phasiinae). Entomol Ger 4:344-348

Kugler J (1980a) New taxa of Tachinidae (Diptera) with a list of the species from Israel and adjacent territories. Isr J Zool 13(1979):27-60

Kugler J (1980b) A new name to replace Ramona Kugler, 1980 (Diptera: Tachinidae). Isr J Zool 14:67

Latreille PA (1804) Tableau méthodique des insectes. In: Société de Naturalistes et d'Agriculteurs, nouveau dictionnaire d'histoire naturelle, appliquée aux arts, principalement à l'agriculture et à l'économie rurale et domestique. Pp. 129-200. Tome XXIV [Section 3]: Tableaux méthodiques d'histoire naturelle. Déterville, Paris, p $84+4+85+238+18+34$

Latreille PA (1805) Histoire naturelle, genérale et particuliere, des crustacés et des insectes. Ouvrage faisant suite aux oeuvres de Leclerc de Buffon, et partie du cours complet d'histoire naturelle rédigé par C.S. Sonnini, membre de plusieurs Sociétés savantes. Tome quatorzième. F. Dufart, Paris, p 432

Linnaeus C (1758) Systema naturae per regna tria naturae, secundum classes, ordines, genera, species, cum caracteribus, differentiis, synonymis, locis, vol 1, 10th edn. L. Salvii, Holmiae [= Stockholm], p 824

Lioy P (1864) I ditteri distribuiti secondo un nuovo metodo di classificazione naturale. Atti Ist veneto Sci, Ser. 3 9:1311-1352

Loew H (1844) Zur Kenntnis der Ocyptera-Arten. Stettin Entomol Zeit 5:226-240 266-269

Loew H (1845) Noch einige Bemerkungen uber die Gattung Ocyptera. Stettin Entomol Zeit 6:170-183

Loew H (1852) Bericht über die zur Bekanntmachung geeigneten Verhandlungen der Königl. Preufs. Akad Wiss Berlin 1852:658-661

Loew H (1871) Beschreibung europäischer Dipteren. Zweiter Band. Systematische Beschreibung der bekannten europäischen zweiflügeligen Insecten. Von Johann Wilhelm Meigen. Neunter Theil oder dritter Supplementband. H.W. Schmidt, Halle, p viii + 319

Macquart J (1834) Insectes diptères du nord de la France. Athéricères: créophiles, oestrides, myopaires, conopsaires, scénopiniens, céphalopsides. Mem Soc Sci Agric Lille 1833:137-368

Macquart J (1845) Nouvelles observations sur les insectes diptères de la tribu des tachinaires. Ann Soc Entomol Fr, Sér. 2 3:237-296

Macquart J (1848) Nouvelles observations sur les diptères d'Europe de la tribu des tachinaires. (Suite.) Ann Soc Entomol Fr, Sér. 2 6:85-138

Macquart J (1851a) Nouvelles observations sur les diptères d'Europe de la tribu des tachinaires. Suite. Ann Soc Entomol Fr, Sér 2 8(1850):437-492

Macquart J (1851b) Diptères exotiques nouveaux ou peu connus. Suite du 4.E supplément publié dans les Mémoires de 1849. Mem Soc Sci Agric Lille 1850:134-294

Macquart PJM (1849) Huitième ordre. Les Diptères. In: Exploration scientifique de l'Algérie pendant les années 1840, 1841, 1842. Lucas, P. H. (Ed). Sciences physiques, Zoologie III. Histoire naturelle des animaux articulés. Troisième partie. Insectes. Bertrand, Paris, pp 1-527 
Macquart PJM (1850) Nouvelles observations sur les dipteres d'Europe de la tribu des Tachinaires (suite) [concl.] Ann Soc Entomol Fr, Sér 2 7(1849):357-418

Meigen JW (1800) Nouvelle classification des mouches à deux ailes (Diptera L.) d'après un plan tout nouveau. J.J. Fuchs, Paris, p 40

Meigen JW (1803) Versuch einer neuen GattungsEintheilung der europäischen zweiflügligen Insekten. Mag Insekt 2:259-281

Meigen JW (1824) Systematische Beschreibung der bekannten europäischen zweiflügeligen Insekten. Vierter Theil. Schulz-Wundermann, Hamm, p xii + 428

Meigen JW (1826) Systematische Beschreibung der bekannten europäischen zweiflügeligen Insekten. Fünfter Theil. Schulz, Hamm, p xii + 412

Meigen JW (1830) Systematische Beschreibung der bekannten europäischen zweiflügeligen Insekten. Sechster Theil. Schulz, Hamm, p xi + 401

Meigen JW (1838) Systematische Beschreibung der bekannten europäischen zweiflügeligen Insekten. Siebenter Theil oder Supplementband. Schulz, Hamm, p xii +434

Mesnil LP (1939) Essai sur les tachinaires (Larvaevoridae). Monogr Cent Nat Rech Agron 7:1-67

Mesnil LP (1946) Revision des Phorocerini de l'Ancien Monde (Larvaevoridae). Encyc Entomol Série B Mémoires et Notes II Diptera 10:37-80

Mesnil LP (1949) Essai de révision des espèces du genre Drino RobineauDesvoidy Sturmiinae a oeufs macrotypes. Bull Insti R Sci Nat Belg 25(42):1-38

Mesnil LP (1953a) A new tachinid parasite of an embiopteron. Proc R Entomol Soc Lond Series B Taxonomy 22:145-146

Mesnil LP (1953b) Nouveaux tachinaires d'Orient. (1re partie). Bull Insti R Sci Nat Belg 89:85-114

Mesnil LP (1954) Genres Actia Robineau-Desvoidy et voisins (Diptera Brachycera Calyptratae). Exploration du Parc National Albert, Mission GF de Witte (19331935) $81: 1-41$

Mesnil LP (1955) 64g. Larvaevorinae (Tachininae). Flieg Palaearkt Reg 10(Lieferung 186):417-464

Mesnil LP (1956) 64g. Larvaevorinae (Tachininae). Flieg Palaearkt Reg 10(Lieferung 192):513-554

Mesnil LP (1960) Note préliminaire sur les Siphona Meig. (Dipt. Tachinidae) d'Europe et du bassin méditerranéen. Bull Ann Soc R Entomol Belg 96:187-192

Mesnil LP (1962) 64g. Larvaevorinae (Tachininae). Flieg Palaearkt Reg 10(Lieferung 224):753-800

Mesnil LP (1970a) 64g. Larvaevorinae (Tachininae). Flieg Palaearkt Reg 10(Lieferung 281):1-1435

Mesnil LP (1970b) Description de nouveaux tachinaires de I'Ancien Monde, et notes synonymiques (Diptera, Tachinidae). Mushi 44:89-123

Mik J (1891) Dipterologische Miscellen. XIX. Wien Entomol Zeit 10:189-194

O’Hara JE (2008) Tachinid flies (Diptera: Tachinidae). In: Capinera JL (ed) Encyclopedia of Entomology, 2nd edn. Springer, Dordrecht, pp 3675-3686

O'Hara JE (2009) Resurrection of the name Pachycheta Portschinsky for a genus of Tachinidae (Diptera). Zootaxa 1989:66-68

O'Hara JE (2013) History of tachinid classification (Diptera, Tachinidae). ZooKeys 316:1-34

O'Hara JE (2016) World genera of the Tachinidae (Diptera) and their regional occurrence. Version 9.0. PDF document, 93 pp. Available from: http://www. nadsdiptera.org/Tach/WorldTachs/Genera/Gentach_ver9.pdf. Accessed 12 Feb 2018

O'Hara JE, Cerretti P (2016) Annotated catalogue of the Tachinidae (Insecta, Diptera) of the Afrotropical region, with the description of seven new genera. ZooKeys 575:1-344. https://doi.org/10.3897/zookeys.575.6072

O'Hara JE, Shima H, Zhang C (2009) Annotated catalogue of the Tachinidae (Insecta: Diptera) of China. Zootaxa 2190:1-236

Pape T, Blagoderov V, Mostovski MB (2011) Order Diptera Linnaeus, 1758. In: Zhang, Z.Q. (Ed.), animal biodiversity: an outline of higher-level classification and survey of taxonomic richness. Zootaxa 3148:222-229

Pape T, Thompson FC (2017) Systema Dipterorum. Version 2.0. Available from: http://www.diptera.org/ . Accessed 1 Dec 2017

Perris E (1847) Lettre de M. Edouard Perris a M. M sur une excursion dans les Grandes Landes. Mem Acad Sci Belles-Lett Lyon 2:433-506

Perris E (1852) Seconde excursion dans les Grandes-Landes. Ann Soc Linn Lyon 1850/1852:145-216

Richter VA (2001) A new genus and species of tachinid flies (Diptera: Tachinidae) from Iran. Int J Dipterol Res 12:25-28

Robineau-Desvoidy JB (1830) Essai sur les myodaires. Mém. prés. div. Sav Acad Sci Inst Fr, Sér. 2 2:1-813

Robineau-Desvoidy JB (1844) Études sur les myodaires des environs de Paris. Ann Soc Entomol Fr, Sér. 2 2:5-38
Robineau-Desvoidy JB (1850) Myodaires des environs de Paris. Ann Soc Entomol Fr, Sér. 2 8:183-209

Robineau-Desvoidy JB (1851) Myodaires des environs de Paris. Ann Soc Entomol Fr, Sér. 2 9:177-190

Robineau-Desvoidy JB (1853) Sur les eclosions de plusieurs especes de Dipteres, obtenues par le docteur Moret, Medicin a Auxerre. Bull Soc Sci Hist Nat Yonne 7:531-536

Robineau-Desvoidy JB (1863a) Histoire naturelle des diptères des environs de Paris. Tome premier. V. Masson et fils, Paris; F. Wagner, Leipzig, and Williams and Norgate, London, p 1143

Robineau-Desvoidy JB (1863b). Histoire naturelle des diptères des environs de Paris. Tome second. Victor Masson et fils, Paris; Franz Wagner, Leipzig, and Williams and Norgate, London, p 920

Rohdendorf BB (1924) Eine neue Tachiniden-Gattung aus Turkestan. Zool Anz 58:228-231

Rohdendorf BB (1928) Beiträge zur Kenntnis der Salmacia- (Gonia-) Gruppe. (Diptera, Tachinidae). Zool Anz 78:97-102

Rohdendorf BB (1931) Records of Tachinidae (Larvaevoridae), with new African species (Dipt.) Ann Mag Nat Hist, Ser. 10 8:347-351

Rondani C (1845) Descrizione di due generi nuovi di insetti ditteri. Memoria duodecima per servire alla ditterologia italiana. Nuovi Ann Sci Nat Bologna, Ser. 3 2:25-36

Rondani C (1850) Osservazioni sopra alquante specie di esapodi ditteri del Museo Torinese. Nuovi Ann Sci Nat Bologna, Ser. 3 2:165-197

Rondani C (1856) Dipterologiae Italicae prodromus. Vol: I. Genera Italica ordinis Dipterorum ordinatim disposita et distincta et in familias et stirpes aggregata. A. Stocchi [as "Stoccih"], Parmae [= Parma], p 226

Rondani C (1859) Dipterologiae Italicae prodromus. Vol: III. Species Italicae ordinis Dipterorum in genera characteribus definita, ordinatim collectae, methodo analitica distinctae, et novis vel minus cognitis descriptis. Pars secunda. Muscidae. Siphoninae et (partim) Tachininae. A. Stocchi, Parmae [= Parma], p 243

Rondani C (1861a) Dipterologiae Italicae prodromus. Vol. IV. Species Italicae ordinis Dipterorum in genera characteribus definita, ordinatim collectae, methodo analatica distinctae, et novis vel minus cognitis descriptis. Pars tertia. Muscidae. Tachininarum complementum. A. Stocchi, Parmae [= Parma], p 174

Rondani C (1861b) Species europeae generis Phasiae Latreillei observatae et distinctae. Atti Soc Ital Sci Nat Mus Civ Stor Nat Milano 3:205-220

Rondani C (1865) Diptera Italica non vel minus cognita descripta vel annotata observationibus nonnullis additis. Fasc. II. Muscidae. Atti Soc Ital Sci. Nat Mus Civ Stor Nat Milano 8:193-231

Sabrosky CW, Arnaud PH Jr (1965) Family Tachinidae (Larvaevoridae). In: Stone A, Sabrosky CW, Wirth WW, Foote RH, Coulson JR (eds) A catalog of the Diptera of America north of Mexico. United States Department of Agriculture, pp 961-1108 Agriculture Handbook 276. iv + 1696 pp

Speiser P (1910) Cyclorapha, Aschiza. In: Sjöstedt Y (ed) Wissenschaftliche Ergebnisse der schwedischen zoologischen expedition nach dem Kilimandjaro, dem Meru und den umgebenden Massaisteppen, pp 113-198 Deutsch-Ostafrikas 1905-1906 unter Leitung von Prof. Dr. Yngve Sjöstedt. 2. Band [Abteilung] 10 (Diptera). P. Palmquist, Stockholm. 202 pp

Steyskal GC, El-Bialy S (1967) A list of Egyptian Diptera with a bibliography and key to families. Min Agric Tech Bull 3:12-18

Stireman JO, O'Hara JE, Wood DM (2006) Tachinidae: evolution, behavior, and ecology. Annu Rev Entomol 51:525-555

Strobl G (1906) Spanische Dipteren. II. Beitrag. Mem Real Soc Esp Hist Nat 3(1905):271-422

Tooker JF, Hauser M, Hanks LM (2006) Floral host plants of Syrphidae and Tachinidae (Diptera) of Central Illinois. Ann Entomol Soc Am 99(1):96-112

Townsend CHT (1912) A readjustment of muscoid names. Proc Entomol Soc Wash 14:45-53

Townsend CHT (1916) Designations of muscoid genotypes, with new genera and species. Insecutor Inscit Menstr 4:4-12

Townsend CHT (1921) Some new muscoid genera ancient and recent. Insecutor Inscit Menstr 9:132-134

Townsend CHT (1926a) New Holarctic Muscoidea (Diptera). Insecutor Inscit. Menstr 14:24-41

Townsend CHT (1926b) Fauna sumatrensis. (Beitrag Nr. 25). Diptera Muscoidea II. Suppl Ent 14:14-42

Townsend CHT (1927) Fauna sumatrensis. (Beitrag Nr. 50). Diptera Muscoidea III. Suppl Ent 16:56-76

Townsend CHT (1932) Notes on old-world oestromuscoid types. Part II. Ann Mag Nat Hist, Ser. 10 9:33-57

Townsend CHT (1933) New genera and species of old world oestromuscoid flies. J NY Entomol Soc 40(1932):439-479 
van der Wulp FM (1893) Eenige Javaansche Tachininen. Tijdschr Entomol 36:159-188 Verbeke J (1970) In: Hanström B (ed) Diptera (Brachycera): Tachinidae (excl. Siphonina). South African animal life. Results of the Lund University expedition in 1950-1951. Almqvist \& Wiksell. the University of California, USA.

Villeneuve J (1911) Notes diptérologiques. Wien Entomol Zeit 30:84-87

Villeneuve J (1913a) Diptères nouveaux du Nord Africain. Deuxième note. Bull Mus Hist Nat, Paris 18(1912):505-511

Villeneuve J (1913b) Myodaires supérieurs de l'Afrique tropicale (1re liste). Rev Zool Afr 3:24-46

Villeneuve J (1916) A contribution to the study of the south African higher Myodarii (Diptera Calyptratae) based mostly on the material in the south African museum. Ann South Afr Museum 15:469-515

Villeneuve J (1920) Étude de quelques myodaires supérieurs (recueillis par le Dr Brauns, à Willowmore, Cap). Rev Zool Afr 8:151-162

Villeneuve J (1923) Myodaires supérieurs d'Egypte inédits (Dipt.) Bull Soc Royale Entomol Égypte 7(1922):88-94

Villeneuve J (1927) Tachinides nouveaux de Formose et du Congo. Rev Zool Afr 15:217-224

Villeneuve J (1929a) Descriptions de diptères égyptiens. Bull Soc Royale Entomol Égypte 12(1928):43-46

Villeneuve J (1929b) Diagnoses de myodaires supérieurs inédits. Bull Ann Soc Entomol Belg 69:99-102

Villeneuve J (1929c) Propos diptérologiques. Bull Ann Soc Entomol Belg 69:181-187

Villeneuve J (1934a) Myodaires supérieurs peu connus ou inédits de la Palestine. Konowia 13:54-57

Villeneuve J (1934b) Myodaires supérieurs inédits d'Afrique. Rev Zool Bot Afr 26: $68-72$

Villeneuve J (1936) Description de deux myodaires supérieurs (Diptera: Trixiini ou Dexiinae?). Bull Soc R Entomol Egypte 20:329-331

Villeneuve J (1937) Notes dipterologiques. Bull Ann Soc Entomol Belg 77:404-408

Villeneuve J (1939) Présentation de quelques myodaires supérieurs inédits. Bull Ann Soc Entomol Belg 79:347-354

Villeneuve J (1942) Espèces inédites de la famille des Larvaevoridae (Dipt.) Bull Soc Entomol Fr 47:133-135

Walker F (1849) List of the specimens of dipterous insects in the collection of the British Museum. Part IV, British Museum (Natural History), London, pp 6891172

Walker F (1853) Insecta Britannica. Diptera, vol II. Lovell Reeve, London, p vi + 297

Walker F (1871) List of Diptera collected in Egypt and Arabia, by J. K. Lord, Esq. with descriptions of the species new to science. Entomologiste 5:255-263

Westwood JO (1840) Order XIII. Diptera Aristotle. (Antliata Fabricius. Halteriptera Clairv.), pp. 125-154. In: Synopsis of the genera of British insects, p 158

Wiedemann CRW (1819) Beschreibung neuer Zweiflügler aus Ostindien und Afrika. Zool Mag 1(3):1-39

Wiedemann CRW (1824) Munus rectoris in Academia Christiana Albertina aditurus analecta entomologica ex Museo Regio Havniensi, Eregio typoguapheo scholarum, Kiliae [= Kiel], p 60

Wiedemann CRW (1830) Aussereuropäische zweiflügelige Insekten. Als Fortsetzung des Meigenschen Werkes. Zweiter Theil. Schulz, Hamm, p xii + 684

Zeller PC (1842) Dipterologische Beytrage. Zweyte Abtheilung. Isis (Oken's), 1842, pp 807-847

Zetterstedt JW (1844) Diptera Scandinaviae. Disposita et descripta. Tomus tertius. Officina Lundbergiana, Lundae [= Lund], pp 895-1280

Zetterstedt JW (1859) Diptera Scandinaviae. Disposita et descripta, vol 13. Officina Lundbergiana, Lundae [= Lund], pp 4943-6190

Zimin LS (1954) Species of the genus Linnaemyia Rob.-Desv. (Diptera, Larvaevoridae) in the fauna of the USSR. Trudy Bot Inst Akad Nauk SSSR 15: 258-282

Zimin LS (1961) A review of the Palaearctic genera and species of the subtribe Peletieriina (Diptera, Larvaevoridae). Trudy Vses Entomol Obshch 48:230-334

\section{Submit your manuscript to a SpringerOpen ${ }^{\circ}$ journal and benefit from:}

- Convenient online submission

- Rigorous peer review

- Open access: articles freely available online

- High visibility within the field

- Retaining the copyright to your article

Submit your next manuscript at $\boldsymbol{\nabla}$ springeropen.com 